\title{
Phänomenologie, Psychologie, Erkenntnistheorie.
}

Von Theodor Elsenhans.

Inhalt. Einleitung. - A. Phänomenologie und Psychologie. - I. Allgemeine Umgrenzung der Phänomenologie. - II. Die "Wesenserschauung.“ - III. Die „reinen Gegebenheiten" und die Analogie der Mathematik. IV. Das Verhăltnis der Phänomenologie zur empirischen Psychologie, der Beschreibung zur begrifflichen Bearbeitung. - V. Die Einzelanwendung der Phänomenologie als deskriptiver Psychologie. - Ergebnis. - B. Phänomenologie und Erkenntnistheorie. - I. Die letzte Rechtsquelle aller Erkenntnis. - II. Die Evidenz. - III. Reflexion und Selbstbeobachtung und die Überwindung des Zweifels an ihren Ergebnissen. - IV. Die Frage des Kriteriums und seines Verbältnisses zur Evidenz. - V. Die Voraussetzungslosigkeit der Phänomenologie in ihrem Verhältnis zur Erkenntnistheorie. - VI. Der Begriff der Intention und die "Bildertheorie.“ - VII. Die Notwendigkeit eines im "praktischen Realismus" gegebenen voraussetzungslosen Ausgangspunktes.

In die grundsätzlichen Erörterungen der Psychologie der Gegenwart ist die Phänomenologie mit steigendem Erfolge eingetreten. Die bedeutenden Leistungen, die sie aufzuweisen hat, und die wissenschaftlichen Ansprüche, die sie erhebt, nötigen, sofern in die Wirrnis der augenblicklichen Lage einige Klarheit kommen soll, zu einer Auseinandersetzung mit ihr, wobei neben der Psychologie die Erkenntnistheorie und die Logik wesentlich interessiert sind. In solchen Fällen ist es zweckmässig, aus der Vielheit der Erscheinungen diejenige herauszugreifen, in welcher die betreffende Richtung ihre schärfste Ausprägung gefunden hat. Für Edmund Husserl trifft nicht bloss dieser Gesichtspunkt zu, sondern er kann, wenn es auch an Andeutungen anderer und an Anläufen verschiedener Art vorher nicht gefehlt hat, als der eigentliche Schöpfer dieser Richtung betrachtet werden. Seine neueste Schrift, die "Ideen zu einer reinen Phänomenologie und phänomenologischen Philosophie" enthält ein vollständiges Programm derselben mit eingehender wissenschaftlicher 
Ausführung und Begründung. $\left.{ }^{1}\right)$ Die Zeitschrift, welche diese Abhandlung eröffnet, das „Jahrbuch für Philosophie und phänomenologische Forschung“ will aber überhaupt dem in weiten Kreisen sich bekundenden Verlangen entgegenkommen, „die Eigenart phänoInenologischer Methode und die Tragweite ihrer Leistungen kennen zu lernen." Zur Beurteilung der letzteren sind also auch die weiteren ivisher in dieser Zeitschrift erschienenen Abhandlungen von Pfänder, Scheler, Geiger und Reinach beizuziehen, die eine willkommene Illustration der Anwendung dieser Methode auf bestimmte Einzelfragen liefern. Husserls eigene frühere Arbeiten, insbesondëre seine "logischen Untersuchungen" stimmen mit der vollkommen ausgebildeten „Phänomenologie" der "Ideen" nicht mehr durchaus überein. Für die logischen Untersuchungen ist angesichts der Unmöglichkeit, „das alte Werk ganz und gar auf das Niveau der Ideen zu erheben,“ eine Umarbeitung gewählt worden, "die den Leser in bewusster Weise emporleitet, und zwar so, dass in der letzten Untersuchung im wesentlichen die Stufe der "Ideen" erreicht ist und in ihr die trüher mit in den Kauf genommenen Unklarheiten und Halbheiten

1) Jahrbuch für Phänomenologie und phänomenologische Forschung, heransgegeben von Edmund Husserl. Erster Band, Teil 1. Ideen zu einer reinen Phänomenologie und phänomenologischen Philosophie. Einleitung. 1. Buch. Allgemeine Einführung in die, reine Phänomenologie von E. Husserl. Zur Psychologie der Gesinnungen von Alexander Pfänder. Teil 2. Der Formalismus in der Ethik und die materiale Wertethik von Max Scheler. Beiträge zur Phănomenologie des ästhetischen Genusses von Moritz Geiger. Die apriorischen Grundlagen des bürgerlichen Rechtes von Adolf Reinach. Dazn das eben in zweiter Auflage erschienene frühere Hauptwerk: Edmund Husserl, Logische Untersuchungen 2 Bände 2. A. 1913. (zitiert: Log. Unt.) Ferner kommen folgende kleinere Abhandlungen Husserls in Betracht: Bericht über deutsche Schriften zur Logik in den Jahren 1895-99, Archiv für systematische Philosophie, Bd. X (1903) S. 397-400 und Philosophie als strenge Wissenschaft, Logos Band I (1910-11) S. 316-318. Endlich seien von älteren Forschern, die Husserls Phänomenologie nahe stehen, noch die folgenden genannt: W. Dilthey, Ideen über eine beschreibende und zergliedernde Psychologie, Sitzungsberichte der Kgl. preuss. Akad. der Wissensch. zn Berlin 1894, S. 1309-1407. C. Stumpf, Erscheinungen und psychische Funktionen, ebenda 1906. W. Dilthey, Studien zor Grundlegung der Geisteswissenschaften 1905. Th. Lipps, Inhalt und Gegenstand: Psychologle und Logik. Sitzangsberichte der philosoph. philol. und der hist. Kl. der K. bayr. Al. der Wissensch. 1905 S. 511-669. Ders., Bewusstsein and Gegen-. stande, Psychologische Untersuchungen herausg. von Theodor Lipps I Bd 1907 S. 1-203. Ders, Die „Erscheinungen*, ebenda S. $523 \mathrm{ff}$. 
einsichtig geklärt erscheinen, " $)$ Dagegen steht die Abhandlung „Philosophie als strenge Wissenschaft" bereits völlig auf dem Standpunkt der "Ideen" und hat ihre programmatische Bedeutung darin, dass sie unter schroffer Betonung der Unwissenschaftlichkeit aller bisherigen Philosophie eben der Phänomenologie die Aufgabe zuweist, der Philosophie eine streng wissenschaftliche Grundlage zu schaffen.

\section{A) Phănomenologie und Psychologie.}

\section{Allgemeine Umgrenzung der Phänomenologie.}

Für das Verständnis der Phänomenologie in dem jetzt von Husserl vertretenen Sinne ist nun in erster Linie die Abgrenzung gegenüber der deskriptiven Psychologie massgebend. Der Unterschied zwischen beiden tritt schon ätusserlich darin hervor, dass die Phänomenologie sich einer eigenen von den herkömmlichen Bezeichnungen der Psychologie völlig abweichenden Terminologie bedient. Dadurch ist insbesondere die Lektüre der "Ideen" ausserordentlich erschwert. Man mag es beklagen, dass hierdurch die Diskussion über diese zentralen Fragen mit einer neuen den Terminis nach zum Teil an Aristoteles und die Scholastiker sich anlehnenden, dem Sinne nach neuen Schulsprache belastet und das Streben nach einer einheitlichen Terminologie an einem wichtigen Punkte gehemmt wird, man mag unter diesen Umständen es ferner begreiflich finden, dass der Verfasser fast jedem seiner Kritiker gegenüber Missverständnisse zu beklagen hat, ${ }^{2}$ ) aber man wird demjenigen nicht ohne weiteres das Recht $z \mathfrak{u}$ einer solchen individuellen Formgebung für seine Gedanken bestreiten können, der Neues und Wertvolles zu bringen hat und in der Begriffssprache der augenblicklichen Wissenschaft nicht die mit seinen Begriffen sich deckenden Ausdrücke findet. Die Prüfung, ob diese Voraussetzungen zutreffen, wird allerdings um so schärfer sein müssen, wenn noch hinzukommt, dass, wie Husserl sagt, „in der anfangenden Phänomenologie alle Begriffe, bzw. Termini, in gewisser Weise in Fluss bleiben müssen, immerfort auf dem Sprunge, sich gemäss den Fortschritten der Bewusstseins-Analyse und der Erkenntnis neuer phänomenologischer Schich-

1) Log. Unt. Vorwort zur 2. Aufl.

2) So gegenüber Külpe, A. Messer, J. Colın, "Ideen" S. 11, 158. An ersterer Stelle heisst es z.B. das Missverstandnis sei ein so vollkommenes, ndass vom Sinne der eigenen Feststellungen nichts mehr tibrig bleibt." 
tungen innerhalb des zunächst in ungeschiedener Einheit Erschauten zu differenzieren. " ${ }^{1}$ ) Die in einer Sonderterminologie an sich schon liegenden Schwierigkeiten werden dadurch in erheblichem Masse gesteigert.

Der Abweichung in der äusseren Form entspricht die inhaltliche Abgrenzung der Phänomenologie gegenüber der deskriptiven Psychologie, und damit ergibt sich zunächst eine negative Bestimmung ihres Begriffes. Die deskriptive Psychologie ist als empirische Psychologie eine Erfahrungswissenschaft d. h. eine Wissenschaft von Tatsachen und von Realitäten, von realen Vorkommnissen, die als solche mit den realen Subjekten, zu denen sie gehören, einer räumlich-zeitlichen Welt sich einordnen. Die Phänomenologie aber hat es nicht mit einzelnen Erfahrungstatsachen zu tun. Sie liefert zwar wesentliche Fundamente für die Psychologie, aber sie ist so wenig selbst.Psychologie, wie die Geometrie Naturwissenschaft ist. Sie will nicht Tatsachen feststellen, sondern - und damit gelangen wir zu einer positiven Bestimmung der Phänomenologie - Wesenserkenntnisse. Sie ist nicht Tatsachenwissenschaft sondern Wesenswissenschaft. Wer diesen Übergang von der psychologischen Tatsache zum reinen "Wesen" d. h. die "eidetische Reduktion" vollzieht, verhält sich damit zu jener Welt der Tatsachen ähnlich wie der Geometer zum Naturforscher. "Der Geometer, der seine Figuren auf die Tafel malt, erzeugt damit faktisch daseiende Striche auf der faktisch daseienden Tafel, Aber sowenig wie sein physisches Erzeugen ist sein Erfahren des Erzeugten, quâ Erfahren, begründend für sein geometrisches Wesensschauen und Wesensdenken. Daher ist es gleich, ob er dabei halluziniert oder nicht, und ob er, statt wirklich zu zeichnen, sich seine Linien und Konstruktionen in eine Phantasiewelt hineinbildet. Ganz anders der Naturforscher. Er beobachtet und experimentiert, d. i. er stellt erfahrungsmässiges Dasein fest, das Erfahren ist für ihn begründender Akt, der nie durch ein blosses Einbilden ersetzbar wäre. " 2)

Zweitens grenzt sich die Phänomenologie der Psychologie gegenüber dadurch $a b$, dass ihre Phänonene irreal sind. Wie der Geometer nicht Wirklichkeiten sondern "ideale Möglichkeiten" erforscht, so sind es nicht reale sondern "transzendental reduzierte Phänomene," mit denen die Phänomenologie sich beschäftigt. Sie ist

1) „Ideen" S. $170 \mathrm{f}$.

2) "Ideen" S. 3 f. 17 
also - damit fassen wir nunmehr ihre bisher festgestellten Merkmale in einer positiven Bestimmung zusammen: - eine Wesenslehre transzendental reiner Erlebnisse.

Eben indem sie Erlebnisse beschreibt, unterscheidet sie sich aber zugleich als deskriptive Wissenschaft von den exakten Wissenschaften. Während z. B. die exakten geometrischen Begriffe als Idealbegriffe, die etwas ausdrücken, was man nicht "sehen" kann, eine von aller Dinggegebenheit unabhängige Bestimmtheit haben, haftet den Beschreibungsbegriffen der Phänomenologie mit Notwendigkeit eine gewisse Unbestimmtheit an. Sie grenzen sich also durch diese ihre „Vagheit," die mit dem Umstand zusammenhängt, dass sie fliessende Sphären ihrer Anwendung haben, ihrerseits wieder der Mathematik gegenüber ab. ${ }^{1}$ )

Die $\mathrm{Phänomenologie} \mathrm{ist} \mathrm{also} \mathrm{noch} \mathrm{genauer} \mathrm{zu} \mathrm{bestimmen} \mathrm{als}$ "deskriptive Wesenslehre reiner Erlebnisse."

\section{Die „Wesenserschauung.“}

Daran schliesst sich nun aber sofort die weitere Frage: auf welches Verfahren gründet sich diese Deskription? Wenn die Wesensverhalte in „unmittelbarer Einsicht erfasst" werden, worin besteht dieses Erfassen?

$\mathrm{Da}$ es sich nicht um die Phänomene als Tatsachen, sondern um ihr Wesen handelt, so ist man zunächst geneigt, dieses „Erfassen“ als eine Aufgabe des Denkens anzusehen, das in Begriffen, Urteilen, Schlüssen sich vollzieht. Auch nach Husserl sind die Ergebnisse der phänomenologischen Erkenntnis in begriffliche Ausdrücke zu fassen und in der weiteren wissenschaftlichen Reflexion logisch streng zu begründen; aber der Erkenntnisinhalt als solcher wird nicht durch das begriffliche Deuken gewonnen, sondern vermittelst der unmittelbaren Intuition, der "Wesenserschaung.“ Auch die durch Gattung und Art bezeichneten Wesensverhältnisse dürfen nicht verwechselt werden mit der rein logischen Subsumption eines niederen Begriffs unter einen höheren. Vielmehr ist das allgemeine Wesen ebenfalls in dem besonderen in einem bestimmten, ebenfalls „in der eidetischen Intuition seiner Eigenart nach zu erfassenden Sinne" enthalten. ${ }^{2}$ )

1) "Ideen" S. $138 \mathrm{ff}$.

2) "Ideen" S. $25 \mathrm{ff}$. 
So lässt sich das Verfahren der Phänomenologie in folgender Weise zusammenfassen: „sie hat sich reine Bewustseinsvorkommnisse exemplarisch vor Augen zu stellen, sie zu vollkommener Klarheit zu bringen, an ihnen innerhalb dieser Klarheit Analyse und Wesenserfassung zu üben, den einsichtigen Wesenszusammenhängen nachzugehen, das jeweils Geschaute in getreu begriffliche Ausdrücke zu fassen, die sich ihren Sinn rein durch das Geschaute, bzw. generell Eingesehene vorschreiben lassen usw. ${ }^{1}$ ).

So ist also die eigentliche Quelle der Wesenserkenntnis die Anschauung. In der "Wesenserschaunng" wird ebenso ein Wesen gegenständlich erfasst, wie in der individuellen oder erfahrenden Anschauung ein individueller Gegenstand erfasst wird. Das Wesen ist in der tat ein "neuartiger Gegenstand, “ der in „originär gebenden Anschauungen" erfasst wird. Husserl selbst findet darin eine gewisse Annäherung an den Positivismus. „Sagt Positivismus", meint er, "soviel wie absolut vorurteilsfreie Gründung aller Wissenschaften auf das Positive, d. i. originär zu Erfassende, dann sind wir die ächten Positivisten. Wir lassen uns in der tat durch keine Autorität das Recht verkümmern, alle Anschauungsarten als gleichwertige Rechtsquellen der Erkenntnis anzuerkennen.“ ${ }^{2}$ )

Sucht man von diesem im Mittelpunkt der Phänomenologie stehenden Begriff der Wesenserschauung, die weder Begriff noch empirische Anschauung sein soll, sich nun eine nähere Vorstellung zu machen, so liegt es nahe, an geschichtliche Anknüpfungen zu denken. Den Vorwurf des Platonischen Realismus weist Husserl als Verwechslung von Gegenstand und Realem, Wirklichkeit und realer Wirklichkeit selbst zurïck. ${ }^{3}$ ) Kants "intuitus originarius" ist auf das "Urwesen“" beschränkt, Fichtes intellektuelle Anschaung ist „ das dem Philosophen angemutete Anschauen seiner selbst im Vollziehen des Aktes, wodurch ihm das Ich entsteht ${ }^{\text {"4)}}$ ), bei Schelling ist sie im Unterschied von der sinnlichen eine Anschaung, in welcher das Produzierende

) „Ideen S. 123.

\% "Ideen" S. $10 \mathrm{f}, 13.38$. S. $288 \mathrm{ff}$.

? "Ideen" S. 40 ff, vgl. dazu Natorp, Allgemeine Psychologie I (1912)

7 Die deutlichste Auseinandersetzung dieses Begriffs in der zweiten Einleitung in die Wissenschaftslehre (SW. I, 463) berührt sich allerdings an einem Punkte, in der Anffassung der Beziehung zwischen intellektueller nnd sinnlicher Anschauung mit Husserl. 
mit dem Produzierten eins und dasselbe ist. " ${ }^{1}$ ) Etwas enger ist die Berührung mit Schopenhauers intuitiver philosophischer Erkenntnis der Ideen, mit seiner Auffașung der Philosophie als eines „Mittleren von Kunst und Wissenschaft", und als einer Sache genialer das Wesen der Dinge unmittelbar erfassender Denker. ${ }^{2}$ )

Von allen diesen geschichtlich dagewesenen Anschauungsbegriffen unterscheidet sich Husserls "Wesenserschauung“ durch die Hervorhebung ihres beschreibenden, ihres "deskriptiven" Charakters und ihrer Objekte als „reiner" oder „phänomenologischer Gegebenheiten." Man trifft überhaupt wohl am schärfsten den Grundcharakter dieser Phänomenologie und ihrer geschichtlicheu Stellung, wenn man die beiden Grundmerkmale zusanmen nimmt: dass sie deskriptiv und dass sie nicht-empirisch zugleich sein will. Sie hat mit den Erfahrungswissenschaften gemein, dass es sich bei ihr un "Gegebenheiten" hạndelt, aber es sind nicht empirische sondern "reine Gegebenheiten“ oder "phänomenologische Gegebenheiten.

III. Die „reinen Gegebenheiten" und die Analogie der Mathematik.

Was es heisst, ein Gegebenes beschreiben, können wir uns innerhalb der sinnlichen Wahrnehmung ganz wohl vorstellen. Doch tritt dabei der Gedanke deutlich hervor, dass wir uns dabei in wesentlichen passiv verhalten, - ein Gedanke, der in der Kantischen' Erkenntnistheorie seinen Ausdruck in der Lehre von dem "afficierenden Gegenstand" findet. Was aber die ,originär gebende“ Wesenserschauung oder "Ideation" von der Wahrnehnung als "originär gebender Erfahrung“ unterscheidet, das ist ja doch nicht selbst wieder in demselben Sinne "gegeben," wie der individuelle Gegenstand, es ist vielmehr durchaus abhängig von unserer „phänomenologischen Einstellung, “ es ist ein Plus, das jedenfalls insofern in höherem Masse von uns selbst stammt, als sein Auftreten nicht wie das des individuellen Gegenstandes durch irgendwelche "Affection“ bedingt ist, sondern ausschliesslich von unserer Einstellung abhängt. Es gehört ja zur Eigenart der Wesensanschauung, dass ihr ein Erscheinen, ein "Sichtigsein" von Individuellem - sei es nun in „Erfahrungsgegebenheiten" oder in blossen „Phantasiegegebenheiten“.

1) Schelling, System des transzendentalen Idealismus 1800 S. 50.

2) Schopenhauer, Neue Paralipomena (Nachlass her. von Grisebach IV. Bd. § $28 \mathrm{ff}$. 
— „zugrunde liegt.“" 1 ) Sie bedient sich aber dieser individuellen Anschauung nur zur Exemplifikation, ohne das Individuelle irgendwie als Wirklichkeit zu setzen. Welchen Sinn hat hier das blosse Beschreiben? Ist es tatsächlich so, dass das „reine Wesen" unabhängig von uns da ist, um von uns dann „erfasst", „beschrieben“. zu werden? Die letztgenannten Ausdrücke, wie auch die Massnahmen der „Ausschaltung“ oder der „Einklammerung“ der ganzen in der „natürlichen Einstellung“ gesetzten, in der Erfahrung wirklich vorgefundenen Welt, nach welcher nur die eigenartige Seinsregion der Phänomenologie übrig bleiben soll, ${ }^{2}$ ) scheinen auf diesen Gedanken hinzuweisen. Aber diese Seinsregion hat ja doch nicht Realität wie die Erfahrungswelt? Aber sie ist doch eine Welt „reiner Gegebenheiten“, eine Welt absoluten Seins, und nicht wir sind es, die sie schaffen. Das Tun des "Wesensforschers" beschränkt sich auf die "phänomenologische Einstellung“, durch welche sich ibm diese Welt „reiner Gegebenheiten“ erschliesst.

Je kühner aber von dieser Erwägung der Aktivität oder Passivität des erkennenden Subjekts aus diese Behauptung einer besonderen „phänomenologischen" Erkenntnis erscheinen muss, desto dringender erhebt sich die Frage: worauf kann sich strenggenommen diese Behauptung gründen? Hier ist es nun ein charakteristisches Moment der Husserlschen Phänomenologie, dass dafür in weitestem Umfange die Analogie der Mathematik, insbesondere der Geometrie massgebend wird. Die Geometrie erscheint als Typus der Wesenswissenschaft gegenüber den Tatsachenwissenschaften. Wie der Geometer so erforscht auch der Phänomenologe nicht Wirklichkeiten, sondern Wesensverhalte. „Geometrie und Phänomenologie als Wissenschaften reiner Essenz kennen keine Feststellungen über reale Existenz". Damit hängt es ja auch zusammen, "dass ibnen klare Fiktionen nicht nur eben so gute, sondern in grossem Umfange bessere Unterlagen bieten, als Gegebenheiten aktueller Wabrnehmung und Erfabrung “ ${ }^{8}$ ). Für beide ist daher auch nicht die Erfahrung, sondern die "Wesenserschauung" der letzten Endes sie begründende Alkt. Es ist wohl kein Zufall, dass innerhalb dieser mathematischen Parallele der Phänomenologie mit dem Fortschritt von den "Logischen Untersuchungen" ${ }^{\prime}$ u den "Ideen" an die Stelle

1) Ideen S. 12.

2) Ideen, S. $52 \mathrm{ff}, 94$.

э Ideen, S. 153. 
der Arithmetik als typischen Beispiels mehr und mehr die Geometrie tritt, deren anschaulicher Charakter der stärkeren Betonung der Wesens erschaung in der "Ideation" mehr entsprach. Jedenfalls aber werden die eigentlichen Schwierigkeiten einer solchen Anschauung des "reinen Wesens ${ }^{\text {“ }}$ eben durch diese Analogie der Geometrie zum Teil verdeckt. Was hier möglich ist, scheint auch auf einem ganz anderen Gebiete möglich zu sein, ohne dass diese Übertragung aus einer grundsätzlichen Übereinstimmung beider Gebiete ausreichend gerechtfertigt würde. In der Geometrie allerdings scheint uns das von Kant zuerst in seiner ganzen Schärfe formulierte Problem gelöst: wie es nicht-empirische Anschauung geben könne. Dürfen wir diese Möglichkeit aber auf ein Gebiet übertragen, wo die mathematische Anschauung völlig versagt? Und sind wir berechtigt, jene eigentümliche Verbindung von "Irrealität" und apodiktischer (und „eidetischer") Notwendigkeit, die der Mathematik zweifellos zukommt, auch der Phänomenologie zuzuschreiben, deren Objekte doch einen ganz anderen Charakter haben? Und wenn man das Problem unseres Anteils an der Erkenntnis auf dem Boden der Mathematik in der von Kant erst nachträglich deutlich herausgearbeiteten Verbindung einer synthetischen Funktion mit der Anschauung gelöst findet, müsste dann nicht der phänomenologisch eingestellte nur beschreibende "Wesensforscher" jede grundsätzliche Analogie dieser Art ablehnen?

Allerdings verkennt Husserl selbst keineswegs den Unterschied zwischen der Phänomenologie als beschreibender und der Mathematik als exakter Wesenswissenschaft. Den „formalen“ mathematischen Disziplinen gegenüber ist sie ohnedies deutlich abgegrenzt, da die Phänomenologie offenbar den "materialen" "Wesens-" oder „eidetischen" Wissenschaften angehört. Aber auch von materialen "eidetischen" Disziplinen, wie der Geometrie, ist sie deutlich zu unterscheiden. Sie kann nicht als eine "Geometrie der. Erlebnisse" konstituiert werden ${ }^{1}$ ). Das Verfahren der letzteren ist nämlich dadurch charakterisiert, dass "eine endliche Anżahl, gegebenenfalls aus dem. Wesen des jeweiligen Gebietes zu schöpfender Begriffe und Sätze die Gesamtheit aller möglichen Gestaltungen des Gebietes in der Weise rein analytischer Notwendigkeit vollständig und eindeutig bestimmt, so dass also in ihm prinzipiell nichts mehr offen bleibt." Eine solche "mathematisch-definite Mannigfaltigkeit"

1) Ideen, S. 133ff. 
kommt der Phänomenologie als deskriptiver Wissenschaft nicht zu. Hat sie z. B. ein Erlebnis der Gattung "dingliche Phantasie" zu beschreiben, so ist das „phänomenologisch Singuläre“ eben „diese Dingphantasie, in der ganzen Fülle ihrer Konkretion, genau so wie sie im Erlebnisfluss dahinfliesst, genau in der Bestimmtheit und Unbestimmtheit, mit der sie ihr Ding bald von den, bald von jenen Seiten zur Erscheinung bringt, genau in der Deutlichkeit oder Verschwommenheit, in der schwankenden Klarheit und intermittierenden Dunkelheit usw., die ihr gerade eigen ist." Wir erfahren in diesem Zusammenhang zugleich mit aller wünschenswerten Klarheit, wie sich der Übergang von der empirischen inneren Wahrnehmung zu der phänomenologischen Wesenserschauung vollzieht. „Nur die Individuation lässt die $\mathrm{Phänomenologie} \mathrm{fallen,} \mathrm{den} \mathrm{ganzen}$ Wesensgehalt aber in der Fülle seiner Konkretion erhebt sie ins eidetische Bewusstsein und nimmt ihn als ideal-identisches Wesen, das sich, wie jedes Wesen, nicht nur hic et nunc, sondern in unzähligen Exemplaren vereinzeln könnte " ${ }^{1}$ ). Wir hören weiter, dass auch beim weiteren Fortschreiten zu Wesen von „höherer Stufe der Spezialität" z. B. zur Beschreibung des gattungsmässigen Wesens von Wahrnehmung überhaupt, von Erinnerung überhaupt, Einfühlung überhaupt, Wollen überhaupt, die Wesenserschauung herrschend bleibt. Es findet keine solche Dependenz der Leistungen in höheren Stufen von denen in den niederen statt, „dass etwa ein systematisches induktives Verfahren methodisch gefordert wäre, ein schrittweises Emporsteigen auf der Stufenleiter der Allgemeinheit.“ Dazu gehört endlich, dass auch „deduktive Theoretisierungen“ von der Phänomenologie ausgeschlossen sind. Mittelbare Schlüsse sind ihr zwar nicht geradewegs versagt; , aber da alle ihre Erkenntnisse deskriptive, der immanenten Sphäre rein angepasste sein sollen, so haben Schlüsse, unanschauliche Verfahrungsweisen jeder Art nur die methodische Bedeutung, uns den Sachen entgegenzuführen, die eine nachkommende direkte Wesenserschauung zu Gegebenheit zu bringen hat."

Erst in diesen Bemerkungen tritt die allumfassende Herrschaft der Anschauung im Gebiete der Phänomenologie mit voller Schärfe hervor. Als wirkliche Erkenntnis gilt in ihr nur das, was durch die Wesenserschauung „zur Gegebenheit gebracht“ wird. Damit

2) Hierzu und zum folgenden: Ideen S. 140f. und besonders die instruktive Ausfithrung Log. Unt. II. S. 439 f. 
ist nunmehr auch zu völliger Evidenz gezeigt, dass die phänomenologische Wesenserschauung nicht produktive Anschauung ist, die in der Betrachtung ihres Gegenstandes dessen Wesen erzeugt, sondern ein Prozess, der im Grade seiner Passivität der blossen sinnlichen Wahrnehmung gleichkommt. Die "reinen Wesen“ sind da, es komnt nur darauf an, dass wir sie "sehen“. Was wir dazu tun, ist nur die "Einstellung". Allerdings betont Husserl selbst gelegentlich die Spontaneität des "originär gebenden Bewusstseins von einem Wesen " oder der "Ideation", während dem sinnlich gebenden, dem erfahrenden Bewusstsein Spontaneität ausserwesentlich sei: der individuelle Gegenstand könne "erscheinen", auffassungsmässig bewusst sein, aber ohne eine spontane „Betätigung" an ihm. Aber diese Auffassung wird näher dahin erläutert, dass bei der Ideation nicht das Wesen, sondern das Bewusstsein von ihm ein Erzeugtes sei, und die Wesenserschauung wird ausdrücklich, um den skeptischen Einwand, das "Wesen" sei eine Fiktion, auszuschliessen, als Analogon des sinnlichen Wahrnehmens und nicht des Einbildens “ bezeichnet ${ }^{1}$ ). Ist es möglich, dieser Gedankenreihe zu folgen, ohne trotz der Verwahrung des Verfassers an "Platonischen Realismus“ zu denken? Aber die Phänomene der "reinen Phänomenologie" sind doch eben als "irreal" charakterisiert? Wir müssen es daber dabei bewenden lassen, dass sie nicht die "Realität" realer Vorkommnisse haben, die mit ihrem wirklichen Dasein der räumlich-zeitlichen Welt eingeordnet sind. Da sie aber weder als "Gegebenheiten" von uns erzeugt, noch etwa als wahre Urteile unter dem Begriff des "Geltens" untergebracht werden können, so muss ihnen doch wenigstens dasjenige Mass von Realität zukommen, das ihnen ermöglicht, von uns vorgefunden $z \mathfrak{u}$ werden, un schauend erfasst werden zu können. Ihre „Exemplifikation“ an empirischen Gegebenheiten ändert daran nichts, eben deshalb, weil die letzteren nur "Exempel“ sind, an denen jene reinen Gegebenheiten "erschaut" werden. Eine solche - wenn auch noch so sehr verdünnte - Realität „reiner Wesen" mag uns aber im Rahmen gewisser Systeme der Vergangenheit verständlich erscheinen: innerhalb des modernen Denkens dürfte sich für sie kaum ein Ort finden, der ihre Aufstellung in dieser Form rechtfertigte. Und doch ist ihre Annahme, wie wir gesehen haben, eine nicht abzuweisende Konsequenz der Phänomenologie.

1) Ideen S. $42 \mathrm{ff}$. 
Die Voraussetzungen dieser beschreibenden Wissenschaft führen, da ihre Objekte jenseits der Erfahrung liegen und doch auch nicht von uns erzeugt werden, mitten in eine Metaphysik ${ }^{1}$ ) hinein, die um so gewagter ist, als ihr eigentlicher Inhalt nicht aus dem allgemein kontrollierbaren Denken, sondern aus einer ebenfalls nichtempirischen Anschauung stammen soll.

Aber eben dieser letztere Punkt bedarf noch einer besonderen Untersuchung. Man könnte immer noch sagen: es existiert tatsächlich ein besonderer Weg, zur Erkenntnis jener "reinen Wesen“ zu gelangen; wer diesen Weg nicht zu gehen weiss, wird natürlích auch die auf diesem Weg gefundenen Erkenntnisse ablehnen zu müssen glauben. In der Tat stellt sich Husserl auf diesen Standpunkt. Der Übergang von der natürlichen zur phänomenologischen Einstellung ist nicht leicht zu vollziehen. Das neue Feld liegt nicht "so ausgebreitet vor unserem Blicke mit Füllen abgehobener Gegebenheiten, dass wir einfach zugreifen und der Möglichkeit sicher sein könnten, sie zu Objekten einer Wissenschaft zu machen, geschweige denn sicher der Methode, nach der hierbei vorzugehen wäre" \%). Um überhaupt. „das Sachfeld des transzendental reinen Bewusstseins in den erfassenden Blick zu bringen", bedarf es „einer mühsamen Blickabwendung von den immerfort bewussten, also mit den neu intendierten glêichsam verflochtenẹ natürlichen Gegebenheiten", wobei auch all' das fehlt, "was uns für die natürliche Gegenstandssphäre zugute kommt, die Vertrautheit durch eingeübte Anschauung, die Gunst ererbter Theoretisierungen und sachgemässer Methoden", das aus mannigfacher Bewährung in Wissenschaft und Praxis folgende Vertrauen. ${ }^{3}$ ) Aber ist es nicht doch merkwürdig, dass diese Möglichkeit einer von allen Irrtïmern der Erfahrung freien Erkenntnis, die, einmal vorhanden, zu absolut notwendigen und allgemeine Anerkennung erzwingenden Ergebnissen führen soll,

1) Man vergleiche z.B. folgende Stelle in den „Ideen" S.94: „.. unseren erfassenden und theoretisch forschenden Blick richten wir auf das reine Bewusstsein in seinem absoluten Eigensein. Also das ist es, was als das gesuchte "nphänomenologische Residuum“". übrig bleibt, übrig bleibt, trotzdem wirdie ganze Welt mit allen Dingen, Lebewesen, Menschen, uns selbst inbegriffen, $n$, ausgeschaltet ${ }^{*}$ " haben. Wir haben eigentlich nichts verloren, aber das gesamte absolute Sein 'gewonnen, das, recht verstanden, alle weltlichen Transzendenzen in sich birgt, sie in sich " knonstituiert" ".

2) Ideen S. 120.

) Ideen S. 121.

Kantstudien $\mathbf{X X}$. 
nicht schon früher verwirklicht wurde und bisher keinerlei Früchte gezeitigt hat? Dieses sonst wohl missbräuchlich angewandte Argument hat hier doch wohl einige Beweiskraft, wo es sich darum handelt, den seit Jahrhunderten geübten und in ihrer Eigenart erkannten, auf das Wesen der Dinge gerichteten Erkenntnistätigkeiteu des Denkens ein neues Verfahren an die Seite zu stellen.

Jedenfalls aber ist die Frage nicht zu umgehen, welchen Ort diese phänomenologische Funktion im "Erlebnisstrom" der Psyche selbst hat? Allerdings: das Wesen der Phänomenologie als eines „reinen Erlebnisses" ist wiederum phänomenologisch zu erkennen ${ }^{1}$ ). Mit dieser Rückbezogenheit auf sich selbst ist die Phänomenologic auch nicht etwa ganz in derselben Lage, wie die Psychologie und und die Logik, die ebenfalls ihr Verfahren auf sich selbst anwenden. Denn um das Wesen der Phänomenologie zu erkennen, muss der Wesensforscher die Anwendung des Erkenntnisverfahrens selbst erst entdecken und erlernen. Unter allen Umständen müsste jedoch die phänomeniologische Funktion auch im "Erlebnisstrom" selbst empirisch vorgefunden werden. Der.Wesensschauung liegt ja immer eine individuelle Anschauung, sei es nun von Erfahrungs- oder von Phantasiegegebenheiten zugrunde. Ein solcher Ausgangspunkt individueller Anschauung müsste auch für die Phänomenologie vorhanden sein; ja er wäre geradezu eine unerlässliche Voraussetzung der phänomenologischen Erkenntnis. Von dieser Erwägung aus ist es aber doch für diese ganze Richtnng des Erkennens nicht unbedenklich, dass so viele Forscher von dieser "Wesenserschauung" bei sich selbst nichts vorfinden können; oder vielmehr, dass sie dic: Funktion, die dieser „Wesenserschauung“ zugeschrieben wird, stets in dem Verfahren der empirischen deskriptiven Psychologie wiederfinden.

Denn darüber ist kein Zweifel, dass einer der schwächsteu Punkte der Phänomenologie in der Vereinigung zweier Behauptungen liegt: der einen, dass es sich dabei um die Erfassung von „Gegebenheiten" handelt, und der andern, dass dieses Verfahren selbst doch von jeglicher Erfahrung losgelöst sein soll. In der Mathematik, deren Analogie unter anderem die Möglichkeit eines solchen Sachverhalts verdeutlichen soll, kann, wie dies Husserl selbst zugibt, keinenfalls von „reinen Gegebenheiten": in demselben Sinne die Rede sein, wie in der Phänomenologie. Dort handelt es sich um Gegenstände, die als „irreale Möglichkeiten" in der Weise "rein analytischer

1) Ideen S. $122 f$. 
Notwendigkeit" beliebig erzeugt werden, hier um Gegenstände, die in erster Linie in der Erfahrung gegeben sind, und die in der Phänomenologie, wenn auch nicht empirisch, so doch "in der ganzen Fülle ihrer Konkretion" erfasst werden sollen. Der Besonderheit eines konkreten Gegebenen gegenüber bleibt es, wir mögen uns "einstellen", wie wir wollen, bei dem durchgreifenden Unterschied, den Kant für alle Zeit am schärfsten mit den Worten charakterisiert hat: „In Ansehung des letzteren (des „Etwas“, das ein „Dasein enthält und der Empfindung korrespondiert"), welches niemals anders auf bestimmte Art, als empirisch gegeben werden kann, können wir nichts a priori haben, als unbestimmte Begriffe der Synthesis möglicher Empfindungen, sofern sie zur Einheit der Apperzeption (in einer möglichen Erfahrung) gehören. In Ansehung der ersteren (der Form der Anschauung in Raum und Zeit) können wir unsere Begriffe in der Anschauung a priori bestimmen, indem wir uns im Raume und der Zeit die Gegenstände selbst durch gleichförmige Synthesis schaffen, indem wir sie bloss als Quanta betrachten." 1) Es heisst den Begriff der Gegebenheit ins Unverständliche erweitern, wenn man ihn auf etwas anwendet, das, obwohl als ein Konkretes vorhanden, doch nicht in der Erfahrung vorfindbar sein soll. Sollte es, um noch einmal in Kants Sprache zu reden, zwischen der ${ }_{n}$ Rezeptivität der Eindrücke“, durch welche uns ein Gegenstand gegeben wird, und der "Spontaneität der Begriffe“, durch welche dieser „im Verhältnis auf jene Vorstellung“ gedacht wird, noch ein Mittleres geben, dem Spontaneität und Gegebenheit zugleich zugeschrieben werden könnte?

\section{Das Verhältnis der Phänomenologie}

zur empirischen Psychologie, der Beschreibung zur begrifflichen Bearbeitung.

$\mathrm{Zu}$ dieser prinzipiellen Unvereinbarkeit der beiden Begriffe kommt nun aber noch die Schwierigkeit, die in dem Verhältnis jener "reinen ${ }^{4}$ zu den empirischen Gegebenheiten und in dem gegenseitigen Verhältnis ihrer begrifflichen Bearbeitung liegt. Über das Verhältnis der Phänomenologie zu der exakten empirischen Psychologie hat sich Husserl besonders in der Abhandlung über die „Philosophie als streuge Wissenschaft" eingehender ausgesprochen. In

3) Kritik der reinen Vernunft in dem Abschnitt: Die Disziplin der reinen Vernunft im dogmatischen Gebrauche, Ausgabe von Kehrbach B. 555. 
der experimentellen Psychologie erfolge die Beschreibung der Erfahrungsgegebenheiten, der mit ihr Hand in Hand gehenden immanenten Analyse und begrifflichen Fassung derselben mittels eines Fonds von Begriffen, deren wissenschaftlicher Wert für alle weiteren methodischen Schritte entscheidend sei. Eine Psychologie, welche die ihre Objekte bestimmenden Begriffe, z. B. die Worte: Wahrnehmung, Erinnerung, Phantasievorstellung, nur in dem vagen, völlig chaotischen Sinne gebrauche, den sie sich irgendwie in der ,Geschichte" des Bewusstseins zugeeignet haben, hätte ebensowenig Anspruch auf Exaktheit, als es eine Physik hätte, die mit den Alltagsbegriffen von schwer, warm, Masse usw. sich begnügte. Als Wissenschaft von den "psychischen Phänomenen" müsse sie, um diese Phänomene in begrifflicher Strenge beschreiben und bestimmen zu können, sich die nötigen strengen Begriffe in methodischer Arbeit zugeeignet haben, d. h. sie setze phänomenologische Analysen der Begriffsinhalte voraus, die sie auf Erfahrung anwendet, die aber selbst „der Erfahrung gegenüber a priori sind“".") Über diesen Grundmangel habe sie nur ihre "naturalistische Einstellung“ hinwegtäuschen können, sowie ihr Eifer, den Naturwissenschaften nachzustreben und im experimentellen Verfahren die Hauptsache zu sehen, während doch das Psychische „Natur“ in einem zweiten ganz anderen Sinne sei ùnd ihrem Wesen nach nur in immanentem Schauen erfasst werden könne.

Nun lehnt aber Husserl die empirische Psychologie als solche keineswegs völlig $a b$. Er lässt sie vielmehr gelten als Wissenschaft der „psychophysischen Einstellung“, in welcher das „Psychische" mit dem gesamten ihm eigenen Wesen Zuordnung zu einem Leibe und zur Einheit der physischen Natur erhält und mit dieser "indirekten naturhaften Objektivität" „als individuelles

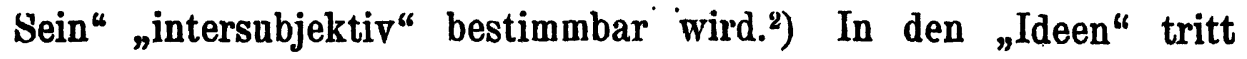
diese Abhängigkeit der empirischen Psychologie von ihrer $\mathrm{Be}-$ ziehung zum Physischen, die Betonung ihres psychophysischen Charakters mehr zurück. Das Erlebnis als solches bildet den erfahrungsmässigen Ausgangspunkt, der zunächst in "natürlicher Einstellung" erfasst und von dem sodann zu der "phänomenologischen Reduktion" übergegangen wird. Indem wir das tun, „verwandeln" wir ja die "Feststellungen" in "exemplarische Fälle

1) Husserl, Philosophie als strenge Wissenschaft S. $306 \mathrm{ff}$.

2) Logos S. $319 \mathrm{ff}$. 
von Wesensallgemeinheiten“, die wir uns dann „im Rahmen reiner Intuition zu eigen machen und systematisch studieren können ".1) Das Bewüsstsein als "Gegebenes der psychologischen Erfahrung ${ }^{4}$. ist Objekt beider Arten der Psychologie, der empirischen in „erfahrungswissenschaftlicher", der "eidetischen Psychologie « in ,: wesenswissenschaftlicher Forschung “. ${ }^{2}$ ) Jedenfalls also kann dasselbe psychische Erlebnis Gegenstand beider Betrachtungsweisen sein. Die "wesenswissenschaftliche" Forschung soll die Grundlage und unerlässliche Voraussetzung der Andern sein.

Aber muss nicht sie selbst auch Rückwirkungen und inhaltliche Berichtigungen von der empirischen Forschung erfahren? Kann sich denn der Wesensforscher völlig einer Verwertung der empirischen Ergebnisse entziehen, die er in Beziehung auf denselben Gegenstand auf anderem Wege ermittelt? Allerdings: die Wesenserkenntnis soll ja von der Tatsachenerkenntnis völlig geschieden sein, "reine Wesenswahrheiten" sollen nicht die mindeste Behauptung über Tatsachen enthalten..$^{3}$ ) Aber es handelt sich doch um wirkliche Geschehnisse, deren empirische Erforschung, anch wenn sie eine vorausgehende Analyse ihres Wesens voraussetzt, im weiteren Verlaufe diese selbst unmöglich unberührt lassen kann? Greifen wir irgend ein Beispiel heraus! „Wir versetzen uns in lebendiger Anschiaung (mag sie auch Einbildung sein) in irgend einen Aktvollzug, etwa in eine Freude über einen frei und fruchtbar ablaufenden theoretischen Gedankengang. Wir vollziehen alle Reduktionen und sehen, was im reinen Wesen der phänomenologischen Sachen liegt. Zunächst also ein Zugewendetsein zu den ablaufenden Gedanken ... usw. " ${ }^{4}$ ) Mit diesem Prozess mischen sich zweifellos, ihn vielfach bedingend und seinem Wesen (in dem gewöhnlichen Sinne des Wortes) nach modifizierend Vorstellungsassoziationen, Lustgefühle und andere der experimentellen und empirisch-deskriptiven Forschung ohne weiteres zugängliche Faktoren. Soll sich nun der Wesensforscher im Augenblick des „immanenten Schauens“ aller der auf diese Gegenstände bezüglichen, ihm bekannten Ergebnisse der empirischen Forschung entäussern? Und wenn die Erfahrungswissenschaft phänomeno-
1) Ideen S. 146.
3) Ideen S. 143.
) Ideen S. 13.
4) Ideen S. 146. 
logisch Geschautes berichtigt, sollte er da diese Berichtigung ignorieren? Natürlich antwortet die Phänomenologie darauf mit Ja, da nach ihrer Grundthese der. Wesensschauung eine aller Erfahrung überlegene Zuverlässigkeit zukommen soll. Aber damit stehen wir wieder unmittelbar vor jener schroffen Scheidung einer Welt "reiner Wesen" und einer Welt der Erfahrungstatsachen, die den Anfang aller Erkenntnis bilden soll und doch zugleich die kühnste aller metaphysischen Hypothesen ist.

So ist es nicht zu verwundern, dass die Husserlsche Phänomenologie trotz des Protestes ihres Urhebers immer wieder mit der empirischen deskriptiven Psychologie verwechselt worden ist. Nicht bloss Husserls eigene frühere Ausdrucksweise hat dazu beigetragen, sondern auch die Scheu der Autoren, die sich in seine logische Gesamtansicht und in seine an die Scholastik allerdings zum Teil gemahnende Terminologie vertieften, diesen Schritt in einen Platon mindestens sich nähernden Begriffsrealismus mit ihm zu tun. Jede wirkliche Ausführung seines phänomemonologischen Programms zeigt auch, dass bei jeder beliebigen bestimmten Aussage über ein Erlebnis jene grundsätzlich scharfe Abgrenzung gegenüber jeglicher Erfahrungswissenșchaft sich nicht halten lässt. Es ist zwar richtig, dass auch die empirisch-induktive Wissenschaft ihre Erkenntnisse keineswegs ausschliesslich aus der Induktion als solcher gewinnt. Abgesehen von den allgemeinen logischen Voraussetzungen alles induktiven Verfahrens, die trotz John Stuart Mill niemals aus dem induktiven Verfahren selbst sich ableiten lassen, operiert auch die empirische Beschreibung psychischer Erlebnisse notwendig mit zunächst noch "vagen" Wortbedeutungen, die sie, da sie doch irgendeinmal beginnen muss, doch nicht immer selbst erst induktiv gewonnen haben kann. Es ist in diesem Zusammenhang durchaus notwendig, sich stets gegenwärtig zu halten, worin der Vorgang der "Beschreibung“ eigentlich besteht. Beschreiben wir einen psychischen Vorgang der Wahrnehmung, der Erinnerung, der Phantasie, der Freude oder des Schmerzes, so bedienen wir uns dabei dieser und anderer Wörter zur Bezeichnung dessen, was wir meinen, in einer Bedeutung, die, wenn auch nur vorläufig und in unbestimmter Weise, doch irgendwie umgrenzt sein muss. Beschreibung ist also immer schon Klassifikation. Die spätere exakte Untersuchung oder eindringendere Analyse mag mancherlei Berichtigung und erst die strengere Begriffsbestimmung liefern; um aber überhaupt beginnen 
zu können, müssen wir aus dem „Strom" psychischen Geschehens einzelne Erlebnisse für unsere Betrachtung ausscheiden und in einer bestimmten Weise bezeichnen.

Man hat daher kein Recht, von einer „bloss beschreibenden Psychologie" in einem Sinne zu reden, als ob in einer solchen bloss Tatsächliches wiedergegeben werden könnte, ohne dass irgendwelche wissenschaftliche Annahmen bereits darin enthalten wären. ${ }^{1}$ ) Jede einen seelischen Vorgang aus dem seelischen Gesammtzusammenhang herausnehmende und damit isolierende Bezeichnung ist. schon eine solche Annahme. Die Bezeichnung selbst stammt auf ihrer vorwissenschaftlichen Stufe, die ja der wissenschaftlichen irgendeinmal vorausgehen muss, aus dem Erwerb des individuellen Sprachgutes, das selbst allerdings als ein Niederschlag von vielerlei Erfahrungen zu gelten hat. Diese vorläufige Begriffsumgrenzung greht dann später mit den durch die Untersuchung des betreffenden Objekts sich ergebenden Modifikationen in die genaue wissenschaftliche Begriffsbestimmung und Klassifikation über, und die Wissen:schaft erfüllt damit eine ihrer wichtigsten Kulturaufgaben, nämlich: :lie in der Sprache niedergelegte Erkenntnis der Wirklicheit durch die Definition der Wortbedeutung fehlerfrei und zuverlässig zu yestalten. Dieser Prozess ist also stets ein Ineinander von Erfahrungen, Beobachtungen, Vergleichangen und begrifflicher Arbeit.

Muss es nun von solchen Erwägungen aus nicht unmöglich rscheinen, dass die $\mathrm{Phänomenologie} \mathrm{ihre} \mathrm{„Beschreibungen“} \mathrm{der}$ "Wesen" vollkommen unabhängig von allen erfahrungsnässigen reststellungen vollzieht? Sollte sich die Behauptung halten lassen, dass alles, was dem Erlebnis rein immanent und reduziert eigentïmlich ist", von aller Natur und Physik und nicht minder von aller Psychologie durch Abgründe getrennt ist?2) Jeder Versuch einer Beschreibung scheint mir das Gegenteil zu beweisen. Wenn Husserl z. B. das Wahrnehmungserlebnis eines blühenden Apfelbaumes

3) zu Diltheys "Ideen über eine beschreibende und zergliedernde Psycho-

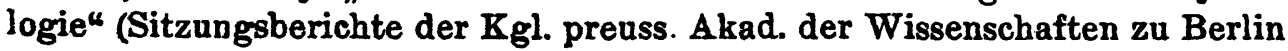
1894, S. 1309-1407) vgl mein Lehrbuch der Psyschologie (1912) S. 48 f.

2) Ideen S. $184 \nabla g l$. hierzu und zum folgenden das ganze Beispiel S. $182 \mathrm{ff}$. Wir sehen auch hier noch vom Begriff der "Intention" ab, der für die hier in Betracht kommenden Seiten der ${ }_{n}$ Beschreibung" nicht wesentlich ist und sich ohne Berücksichtigung der später zu erörternden erkenntnistheoretischen Fragen nicht behandeln lăsst. 
beschreibt und in der "reduzierten Wahrnehmung," d. h. in dem „phänomenologisch reinen Erlebnis, “ als „zu dessen Wesen unaufhebbar gehörig“ "das Wahrgenommene als solches, auszudrücken als "materielles Ding“ „Pflanze,“ „Baum,“ „blühend" usw." findet, so ist doch diese Beschreibung für ihn selbst, wie für den Hörer oder Leser von der empirischen Kenntnis dessen abhängig, was „Pflanze“ „Baum" usw. ist, und diese Kenntnis selbst modifiziert sich mit dem fortschreitenden Wissen und modifiziert auch die Auffassung des "reinen Wesens", das unter Zugrundelegung dieses Wissens beschrieben wird. Es wäre ja auch nicht zu verstehen, wie die phänomenologische Erkenntnis eines Erlebnisses von der fortschreitenden empirischen Erforschung seiner Qualitäten unabhängig sein sollte. Ist dies aber einmal zugegeben, dann ist die Phänomenologie nicht mehr apriorisch, dann fällt der ganze Bau der „reinen Wissenschaft" dahin.

\section{Die Einzelanwendung der Phänomenologie als deskriptiver Psychologie.}

Iṇ der Tat bestätigen es auch alle bisherigen Versuche, die Pbänomenologie auf einzelne Gebiete des Seelenlebens anzuwenden, wic wenig es möglich ịst, von der Wesenserkenntnis eines Erlebnisses jede Begründung aus der Erfahrung fernzuhalten. Gerade das "Jahrbuch für $\mathrm{Phänomenologie} \mathrm{und} \mathrm{phänomenologische} \mathrm{Forschung"“}$ scheint mir vielfaches Zeugnis dafür abzulegen. Wenn z. B. Alexander Pfänder ${ }^{1}$ ) einer Psychologie der Gesinnungen eine „Phänomenologic der Gesinnungen" vorausschicki und dieser die Aufgabe stellt, „vorzudringen bis zur direkten Erfassung des Psychischen selbst und dann eine völlig getreue Beschreibung des psychischen Bestandes selbst zu geben," so zeigt sich schon auf den ersten Seiten, dass diese Beschreibung nicht bloss an "Erlebnisfälle von Gesinnungen“ überhaupt anknüpft, sondern dass auch jede phänomenólogische Aussage über das Wesen der Gesinnungen durch erfahrungsmässige Feststellungen mitbedingt ist. Nicht vermittelst einer geheimnisvollen Wesensschauung wird das Wesen dieser Erscheinungen enthüllt, sondern - ganz wie in jeder andern induktiv-empirischen Vergleichung und Beobachtung, nur mit gröșserer Sorgfalt der begrifflichen Zergliederung - werden aus dem, was an Gesinnungsregungen tatsächlich "vorliegt" oder vorkommt, Schlüsse gezogen. “Auch Max

1) A. Pfänder. Zur Psychologie der Gesinnungen, Jahrbuch I, $325 \mathrm{ff}$. 
Scheler hebt in seiner Abhandlung über den „Formalismus in der Ethik und die materiale Wertethik ${ }^{1}$ ) die Besonderheit der Phänomenologie aller empirischen Wissenschaft gegenüber scharf hervor. Er spricht allerdings von „phänomenologischer Erfahrung“ und nähert sich damit wenigstens im Ausdruck mehr als Husserl der Empirie; aber diese „phänomenologische Erfahrung“" wird dann um so schärfer· aller andersartigen Erfahrung, z. B. der Erfahrung der "natürlichen Weltanschauung der Wissenschaft" gegenüber abgegrenzt. Wir erfahren dabei Genaueres auch über das Verhältnis der mit jener "phänomenologischen Erfahrung" identischen „Wesensschau“ zum Allgemeinbegriff, wie zur Beobachtung und Induktion. Die Wesenheit oder "Washeit," die sie gibt, ist „hierbei als solche weder ein Allgemeines noch ein Individuelles. Das Wesen rot z. B. ist sowohl im Allgemeinbegriff rot, wie in jeder wahrnehmbaren Nuance dieser Farbe mitgegeben." Auch kann das erschaute Wesen "mehr oder weniger gegeben sein - so wie wir einen Gegenstand genauer und weniger genau etwa " "beobachten" "können, oder bald diese, bald jene seiner Züge - sondern es ist entweder „erschaut" und und damit " ${ }_{n}$ selbst" " gegeben (restlos und obne Abzug, weder durch ein "Bild"s noch ein ",Symbol"“ hindurch) oder es ist nicht "n erschaut" " und damit nicht gegeben." "Oder auch: die Wesenheiten und ihre Zusammenhänge sind nämlich " "vor" " aller Erfahrung dieser Art oder auch a priori," gegeben," " die Sätze aber, die in ihnen Erfüllung finden, a priori " "Wahr" "." "Was als Wesen oderZusammenhang solcher erschaut ist, kann also durch Beobachtung und Induktion niemals aufgehoben, nie verbessert oder vervollkommnet werden." 2)

Ganz unmissverständlich ist hier also die "Wesensschau" als ein Absolutes der Erkenntnis bezeichnet, dem gegenüber die ganze vorhergehende und nachfolgende Erforschung desselben "gegebenen" Gegenstandes nichts bedeutet. Wir hahen hier die Frage noch nicht zu erörtern, wie sich diese Annahme, die eigentlich der beliebigen Behauptung eines beliebigen "Wesensforschers" eine völlig unangreifbare Position sichern würde, mit der Frage nach dem Kriterium der Erkenntnis abzufinden vermag. Wir stellen hier nur beilänfig fest, wie nahe sich diese Lehre mit der sonst ganz anders orientierten von Jakob Friedrich Fries berührt. Auch

1) Jahrbuch erster Band, Teil 2, S. 405 ff. zum folgenden vgl. besonders die Ausführungen uber Apriori und Formal überhaupt S. $447 \mathrm{ff}$.

2) a. a. O. S. $447 f$. 
bei letzterem gibt es eine solche „unmittelbare Erkenntnis" absoluter Art im Gebiete der Anschaung. Aber hier ist es die unmittelbare Anschauung durch die Sinne nach ihrem „Dasein im Geiste“, für die es weder Irrtum noch Grade der Gewissheit gibt. ${ }^{1}$ ) Die Motive jedoch, die $\mathrm{zu}$ einem solchen absoluten Ausgangspunkt aller Erkenntnis führen, sind sehr ähnlicher Natur. Wie bei Fries die Unterschiede der Gewissheit und der Irrtum lediglich der "mittelbaren Erkenntnis“, der "wiederbeobachtenden Reflexion" zugeschrieben werden, so hören wir hier, dass nur die "phänomenologische Erfahrung" „die Tatsachen selber und daher unmittelbar" gibt, „d, h, nicht vermittelt durch Symbole, Zeichen, Anweisungen irgendwelcher Art." ${ }^{\text {q)}}$ ) Sie allein gibt z. B. nicht irgendwelche angebbare Bestimmtheiten der Farbe Rot, sondern das Rot selbst. Die Anschauung - das eine Mal die Sinnesanschauung, das andere Mal die Wesenserschaunng - ist in beiden Fällen der Korrektur durch beziehende und vergleichende Tätigkeiten entrückt; nur dass die „phänomenologische Erfahrung“" zugleich jenseits aller "Erfahrung der natürlichen Weltanschauung und der Wissenschaft" liegt und jeglicher Kontrolle der letzteren sich entzieht.

Die Ausführung des Programms zeigt aber auch an dieser Stelle, bei dem Versuche, eine "materiale Wertethik" von der Phänoınenologie aus zu begründen, deutlich die Unmöglichkeit, jede Verwertung erfahrungsmässiger Beobachtung und Vergleichung von dem Einfluss auf die Ergebnisse auszuschliessen. Was Scheler gegenüber den Kantischen k'ormalismus in der Ethik fordert, ist „ein Apriorismus des Emotionalen und eine Scheidung der falschen Ein heit, die bisher zwischen Apriorismus und Rationalismus bestand." „Emotionale Ethik“ im Unterschiede von „rationaler Ethik" sei durchaus nicht notwendig „Empirismus" im Sinne eines Versuches, die sittlichen Werte aus der Beobachtung und Induktion zu gewinnen. "Das Fühlen, das Vorziehen," heisst es da, "das Lieben und Hassen des Geistes hat seinen eigenen apriorischen Gehalt, der von der induktiven Erfahrung so unabhängig ist, wie die reinen Denkgesetze. Und hier wie dort gibt es eine Wesensschau der Akte und ihrer Materien, ihrer Fundierung und ihrer Zusammenhänge. Und hier wie dort gibt es " "Evidenz" " und strengste Exaktheit der phänomenologischen Feststellung." ${ }^{8}$ ) Das Apriori

1) vgl. mein Werk über "Fries und Kant" 1906. II S. $4 \mathrm{ff}$.

2) Ideen S. 449.

3) Scheler a. a. 0. 465. 
ist also hier ein Gegebenes für die Anschauung, dessen Grundmerkmal: Unabhängigkeit von der Erfahrung beibehalten wird, das aber als "Gegebenes" vorgefunden wird, und insofern doch wohl a posteriori ist. ${ }^{1}$ ) Wenn nun der "Tatsachenkreis“, auf den sich eine solche "wertapriorische Materialethik" stützen soll, im einzelnen erörtert wird, wenn wir hören, dass Werte zunächst im Fühlen gegeben sind, dass das „Haben von Werten in keinem Sinne an ein Streben gebunden ist", wenn auf analoge Tatsachen des unwillkürlichen Strebens hingewiesen wird, wenn behauptet wird, dass das „Vorziehen" als Akt völlig zu scheiden ist von der Art seiner Realisierung, dass die Rangordnung der Werte nur "im" Vorziehen und Nachsetzen erfassbar ist, ${ }^{2}$ ) so folgen wir diesen Erörterungen über "Tatsachen", auf welche sich eine materiale Ethik „im Unterschied von Willkürkonstruktionen" stützen soll ${ }^{3}$ ) nicht ohne dauernde Anerkennung des Scharfsinns der Analyse, aber mit wachsendem Staunen darüber, dass der Verfasser dabei glaubt, sich jenseits aller sonstigen empirischen vergleichenden Beobachtung zu befinden und eine "Wesensschau" zu treiben, die durch künftige auf denselben Gegenstand gerichtete empirische Forschung keinerlei Korrektur soll erfahren können. Was wir lesen, sind eindringende deskriptive Analysen, die von Erfahrungstatsacheñ ausgehend, die begrifflichen Grundlagen mit besonderer Sorgfalt, auseinandersetzen.

Dieses Ergebnis wird auch durch die ästhetisch Abhandlung von Moritz Geiger ${ }^{4}$ ) bestätigt und von einer bestimmten Seite her neu belenchtet. Auch hier erfolgt eine prinzipielle Abgrenzung gegenüber der induktiv-empirischen Methode. Das an sich als naheliegend anerkannte induktive Verfahren, bei welchem „alle Arten ästhetischen Geniessens der Reihe nach zu untersuchen, alle Möglichkeiten durchzuprobieren, alle ästhetischen Gefühle $\mathrm{zu}$ analysieren" wären, „um dann zu dem positiven oder negativen Resultat erst am Ende durch die Sichtung der Ergebnisse zu gelangen", wird abgelehnt und dem dann sofort auftretenden Ein-

1) Auch mit diesem Gedanken kommt hier die Phänomenologie der ${ }_{n}$ anthropologischen Kritik der Vernunft ${ }^{\mu}$ von Fries sehr nahe. Vgl. bes. S. 449.

?) a. a. O. S. 434.437 .443 .491$.

8) a. a. O. S. 446.

4) Moritz Geiger, Beiträge zur Phänomenologie des ästhetischen Genusses, Jahrbuch I. Teil II, S. $567 \mathrm{ff}$. 
wand gegenüber, dass man dann sicherlich den entgegengesetzten Weg des deduktiven Verfahrens wählen werde, betont, dass die Induktion nur eine - und zwar eine ganz bestimmte Voraussetzungen für die Möglichkeit ibrer Anwendung in sich schliessende - Methode sei, auf Grund der Tatsachen zu Erkenntnissen zu konmen. Der Satz z. B. „zwei gerade Linien schneiden sich nur in einem Punkte" oder der andere: "Orange liegt zwischen Rot und Gelb auf der Farbenskala" sei - so sicher man zu ihnen durch Feststellung des Gegebenen und nicht durch Spekulation gelangt sei - doch nicht durch Induktion, durch Verallgemeinerung gewonnen. ${ }^{1}$ ) Das mathematische Beispiel scheidet für uns aus, da die Anwendung desselben an der hier doch wohl besonders angreifbaren und von uns früher besprochenen Analogie zwischen Phänomenologie und Mathematik haftet und von „Feststellung des Gegebenen" hier, wenn inbërhaupt, so jedenfalls nur in einen ganz anderen, von allem "Empirischen" sich deutlich abgrenzenden Sinne die Rede sein kann. Dagegen ist das zweite Beispiel gerade für die methodische Prinzipienfrage äusserst instruktiv. Die Einordnung von Orange in die Färbenskala zwischen Rot und Gelb ist natürlich davon abbängig," dass es eine "Farbenskala" wirklich gibt, die selbst doch wohl sicherlich auf dem Wege induktivempirischer Forschung gefunden ist. Das Erlebnis der Qualität "Orange“, das seine Beziehung zu den Erlebnissen „Rot“ und "Gelb" erst ermöglicht, ist natürlich so weuig erst ein Ergebnis der Verallgemeinerung, als es irgend ein anderes Erlebnis ist. Sobald wir aber über jenes Erlebnis und seine Beziehung zu anderen irgend etwas aussagen wollen, - und darum handelt es sich doch immer in der Wissenschaft - so verrät sich in jeder Aussage - und sei es auch nur in der Wortbedeutung der angeblich „reinen Beschreibung“ - die Unmöglichkeit, von aller Verwertung früherer ähnlicher Erfahrungstatsachen völlig abzusehen und alles, was nach induktivem Verfahren aussieht, künstlich auszuschliessen. In unserem Beispiel ist die Aussage nur dadurch möglich, dass in der "Farbenskala" empirische Ergebnisse schon vorliegen, in die ein neues Erlebnis eingeordnet wird. Wir wiederholen: es ist durchaus zuzngeben, dass die Induktion mancherlei Voraussetzungen in sich schliesst, die sie selbst nicht beweisen kann, wir betonen ferner, dass die Induktion, mehr als

1) M. Geiger a. a. O. S. 571 f. 
es nach der oben gegebenen Schilderung ihres Verfahrens wohl scheinen möchte, meist schon in der Fragestellung, in der Regel auch während des Ganges der Untersuchung sich inhaltlich-begrifflicher Elemente bedient, die für das Ergebnis von Bedeutung sind, ohne selbst aus Einzeldaten als deren Verallgemeinerung erst abgeleitet zu sein. Dies ändert aber nichts an der Tatsache, dass in jede derartige „Feststellung“ über Gegebenes frühere Erfahrungen und Ergebnisse früherer Forschungen mit eingehen. Wie wenig es möglich ist, diese Einmischung der Empirie von der phänomenologischen Beschreibung der einzelnen Frlebnisse fernzuhalten, das zeigen auch die weiteren Einzelausführungen Geigers. Bei der Unterscheidung von ästhetischem Gefallen und ästhetischem Genuss beruft er sich darauf, dass "wer unvoreingenommen an die Tatsachen herangehe“", - dazu gehört doch wohl, dass man die verschiedenen in der Erfahrung vorliegenden Tatsachen vergleichend sich vergegenwärtigt - werde von der gewöhnlich behaupteten Identität von Gefallen und Geniessen nichts bemerken. ${ }^{1}$ ) Die Schwierigkeiten in der begrifflichen Abgrenzung des ästhetischen Genusses von anderen Genüssen werden zunächst darauf zurückgeführt, dass zwei Probleme häufig mit einander vermengt werden: das wertästhetisehe Problem der Scheidung des berechtigten und des unberechtigten ästhetischen Genusses und das deskriptive Problem der Scheidung des ästhetischen und des ausserästhetischen Genusses. Die Bearbeitung des letzteren Problems als einer "rein phänomenologischen Frage" verläuft aber fast durchaus in den Formen der vergleichenden Beobachtung. Der Verfasser erinnert an einzelne Genusserlebnisse und an andere zu. ihnen im Gegensatz stehende Erlebnisse, z. B. der Freude, er leitet an zur Vergleichung und Beobachtung derselben und sucht durch begriffliche Zergliederung des Beobachteten und Verglichenen. zur Erkenntnis ihres "Wesens" zu führen. ${ }^{2}$ )

Wesentlich stärker als in der letzterwähnten Arbeit wird. der apriorische Charakter der Phänomenologie betont in der rechtsphilosophischen Abhandlung von Reinach. ${ }^{3}$ ) Trotzdem scheint mir auch hier die angestrebte Apriorität der Sätze, die von den. rechtlichen Gebilden gelten sollen, sofern sie an den "schlichten.

1) a. a. O. S. $573 f$.

2) a. a. O. S. $584 \mathrm{ff}$.

8) Adolf Reinach, Die apriorischen Grundlagen des bürgerlichen Rechtes. Jahrbuch II S. $685 \mathrm{ff}$. 
Tatsachen orientiert" sein will, jene von Husserl vertretene starre Scheidung des Phänomenologischen und des Empirischen auszuschliessen. Schon das erste aus dem "grossen Gebiete der apriorischen Rechtslehre " angeführte Beispiel lässt darüber keinen Zweifel. Der Vorgang des "Versprechens" wird in seiner Eigenart, seinem Verlaufe, seinen Bestandteilen, seinen Sondermerkmalen verfolgt. Es ist von der eigentümlichen Verbindung die Rede, welche das Versprechen zwischen zwei Personen schafft, von der Dauer dieser Verbindung, von dem darin enthaltenen Anspruch, von dem Träger desselben u. s. w. ${ }^{1}$ ) Auch wer bei der Lektüre dieser Ausführungen die Sonderstellung der "spezifisch-rechtlichen Grundbegriffe", für welche der Verfasser ebenfalls die Analogie der mathematischen Gesetze in Anwendung bringt, nicht aus dem Auge lässt, wird sich dem Eindruck nicht entziehen können, dass der Leser hier aufgefordert wird, sich die verschiedenen Fälle zu vergegenwärtigen, in denen er selbst Versprechen erlebt oder beobachtet hat, um aus diesen Fällen seine Erkenntnis des Wesens des Versprechens abzuleiten und an der Hand der eigenen Erfahrung die Ergebnisse des Verfasserers zu bestätigen. Im Sinne der phänomenologischen Methode allerdings sollte nur irgend ein Einzelfall der "Exemplifikation" dienen und an diesem die Wesenserfassung geübt werden. Die phänomenologische Methode muss aber doch darauf rechnen, dass der Leser die ihm vorgeführten Fälle nacherlebt, um von der Richtigkeit der Beschreibung überzeugt za werden. Vergegenwärtigen wir uns aber den Prozess, der sich dabei im Leser abspielt, so erweist es sich als völlig unmöglich, die Verwertung der in der Erinnerung vorliegenden Einzelfälle des betreffenden Erlebnisses auszuschliessen, an dem sich, der Eigenart des menschlichen Denkens entsprechend, mit Notwendigkeit bereits ein Verallgemeinerungsprozess vollzogen hat. Ebensowenig lässt sich die Möglichkeit zurückweisen, dass das einmal festgestellte Wesen durch spätere Erfahrungen eine Korrektur erfährt, kurz: durch alle Fugen des scheinbar so streng abgeschlossenen Baues der phänomenologischen Methode dringen induktiv-empirische Elemente ein. Allzukuihn ist ja auch das Unterfangen, auf die Feststellung von Tatsachen eine Wissenschaft zu gründen und dabei doch das Verfahren der "Tatsachenwissenschaft" auszuschliessen.

1) a. a. O. S. $692 \mathrm{ff}$. 
So kommen wir von verschiedenen Seiten her zu dem Ergebnis, dass die Phänomenologie, falls sie nicht die entschlossene Wendung zu einer Platonischen Metaphysik nehmen will, trotz allen Protestes tatsächlich doch von der deskriptiven Psychologie im empirischen Sinne sich nicht scheiden lässt.1) Ihr geschichtliches Recht soll ihr dalum nicht geschmälert werden. Sie trat als ein beachtenswerter Faktor erfolgreich ein in den Kampf der gegenwärtigen Wissenschaft um die Stellung đer Psychologie, und ihre Arbeit ist aus Motiven heraus entstanden, denen bleibende Bedeutung zuerkannt werden muss. Mit grosser Klarheit hat Husserl die Schwächen der modernen exakten Psychologie in seiner Abhandlung über „Philosophie als strenge Wissenschaft" herausgehoben und die Notwendigkeit einer "systematischen, das Psychische immanent erforschenden Bewusstseinswissenschaft " betont. Er bestreitet mit Recht die „Exaktheit" einer Psychologie, welche ohne vorausgehende Analyse nur mit rohen Klassenbegriffen wie Wahrnehmung, Phantasieanschauung, Aussage, Rechnen und Verrechnen, Grössenschätzen, Wiedererkennen, Erwarten, Behalten, Vergessen usw. arbeitet, ohne den ihre Objekte bestimmenden Begriffen eine wissenschaftliche Fixierung, eine methodische Bearbeitung angedeihen $\mathrm{zu}$ lassen. ${ }^{2}$ ) $\mathrm{Er}$ hat damit das aus der Psychologie der Zeit immer deutlicher aufwachsende Streben seinerseits gefördert, bei aller Anerkennung der erfolgreichen experimentellen Arbeit der selbständigen Analyse der psychischen Phänomene ihr Eigenrecht $z u$ sichern und der drohenden Verwandlung der Gesamtwissenschaft der Psychologie in ein Spezialgebiet der Naturwissenschaft „dem Widersinn einer Naturalisierung von etwas, dessen Wesen das Sein der Natur ausschliesst ${ }^{\text {“ }}, 3$ ) einen Damm entgegenzusetzen. Es ist daher nicht zu verwundern, dass so manche Psychologen, welche gewisse Unzulänglichkeiten des herrschenden Betriebs erkennen und neben den experimentellen Methoden eine selbständige psychologische Analyse für unerlässlich halten, insbesondere die Schule von Lipps, dessen Lebenswerk durchans in dieser Richtung liegt, sich der Phänomenologie nähern oder sich ausdrücklich ihrer schulmässigen Vertretung anschliessen.

1) vgl. hierzu auch A. Messer, Husserls Phanomenologie in ihrem Verhältnis zur Psychologie. Archiv für Psych. XXII, $117 \mathrm{ff}$.

2) Logos S. 303 f., 307.

9) Logos S. 312. 
Zu bedauern ist nur, dass der Kampf gegen den Erbfeind, den ${ }_{n}$ Psychologismus “, und die Überzeugung, von dessen Folgerungen nur bei völliger Loslösung von aller Empirie sich befreien zu können, den Führer dieser Bewegung in eine Richtung hineingedrängt hat, die, sich jenseits aller Erfahrung stellend, es unternehmen will, in einer weder der Kontrolle der Erfahrungswissenschaft, noch derjenigen des begrifflichen Denkens zugänglichen "Wesenserschauung “ reine Gegebenheiten zu erkennen. Auch diejenigen aber, welche empirische und naturwissenschaftliche Bearbeitung der Psychologie nicht identifizieren, werden nicht bloss der grossen Gedankenarbeit, die in der prinzipiellen Begründung dieser Richtung liegt, ihre Anerkennung nicht versagen; sondern sie werden auch in der Phänomenologie, so wie sie in manchen ausgeführten Arbeiten vorliegt, eine wertvolle Bundesgenossin sehen im Kampf um eine selbständige Stellung der Psychologie im Ganzen der gegenwärtigen Wissenschaft.

\section{B) Phänomenologie und Erkenntnistheorie.}

I. Die letzte Rechtsquelle aller Erkenntnis.

Eine grundsätžliche Erörterung der Stellung der Phẳnomenologie zur Psychologie führt mit Notwendigkeit auf erkenntnistheoretische Fragen. Zwar ist die Phänomenologie nicht selbst Erkenntnistheorie; ja sie ignoriert ausdrücklich „die inhaltlichen und vielgestaltigen Probleme der Möglichkeit der verschiedenen Erkenntnisarten und Erkenntniskorrelationen".1) Aber sowohl die Begründung der zentralen Stellung der Phänomenologie innerhalb des Reiches der Wissenschaft überhaupt als insbesondere die erkenntnistheoretische Bedeutung des Anschauungsprinzips schliessen erkenntnistheoretische Probleme ein. Nach Husserl ist "das u nmittelbare "Sehen" ", nicht bloss das sinnliche, erfahrende Sehen, sondern das Sehen überhaupt als originär gebendes

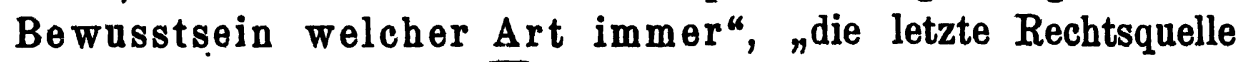
aller vernünftigen Behauptungen". "Rechtgebende Funktion hat sie nur, weil und soweit sie originär gebende ist". ${ }^{2}$ ) Es ist das Prinzip aller Prinzipien: dass jede originär gebende An-

1) Ideen S. 48.

2) Ideen S. $36 \mathrm{f}$. 
schauung eine Rechtsquelle der Erkenntnis sei, dass „alles, was sich uns in der "„Intuition" " originär (sozusagen in seiner leibhaften Wirklichkeit) darbietet, einfach hinzunehmen sei, als was es sich gibt, aber auch nur in den Schranken, in denen es sich da gibt". ${ }^{1}$ ) Jede Aussage, die nichts weiter tut, als solchen Gegebenheiten den angemessenen Ausdruck zu verleihen, ist daher wirklich ,ein absoluter Anfang, im echten Sinne zur Grundlegung berufen, principium". Natürlich kann von diesem Anfang weitergegangen, das Erschaute verarbeitet, Begriffe, Urteile, Schlüsse darauf gegründet werden, aber alle diese späteren Schritte, alle diese ,unanschaulichen Verfahrungsweisen" haben, wie wir früher gehört haben, nur "die methodische Bedeutung“ „uns den Sachen entgegenzuführen, die eine nachkommende Wesenserschauung zur Gegebenheit zu bringen hat". Die Anschauung in dem besonderen Sinne der Phänomenologie bleibt daher auch hier die letzte Rechtsquelle der Erkenntnis. Mit dieser ihrer intuitiven Wesenserkenntnis ist darum die Phänomenologie das „wesentliche eidetische Fundament der Psychologie und der Geisteswissenschaften“. ${ }^{2}$ ) Ja sie umspannt „im Umfange ibrer eidetischen Allgemeinheit" alle Erkenntnisse und Wissenschaften, nämlich, in Hinsicht alles dessen, was an ihnen unmittelbar einsichtig ist". Als angewandte $\mathrm{Phänomenologie} \mathrm{leistet}$ sie „an jeder prinzipiell eigenartigen Wissenschaft" „die letztauswertende Kritik und damit insbesondere die letzte Sinnesbestimmung des ",Seins“" ihrer Gegenstände und die prinzipielle Klärung ihrer Methodik". Es ist daher begreiflich, dass die Phänomenologie ,gleichsam die geheime Sehnsucht der ganzen neuzeitlichen Philosophie ist". Bei Descartes, bei Locke und Hume, bei Kant finden sich ihre Spuren.

Damit tritt die allumfassende Bedeutung der Phänomenologie, wie sie von ihrem Urheber gedacht ist, erst ins rechte Licht. Sie erst liefert die zuverlässigen Grundlagen für die Philosophie und durch sie für die Wissenschaft überhaupt. Das in ihr herrschende Prinzip der "originär gebenden Anschaung" schafft einen Inbegriff sicherer ursprünglicher Erkenntnisse, die, unabhängig von irrtumsfähigen logischen Prozessen oder gar subjektiven Hypothesen, den Ausgangspunkt und zugleich das Kriterium für alle

1) Ideen S. $43 \mathrm{f}$.

2) vgl. hierzu und zum folgenden: Ideen S. 34. $118 \mathrm{f}$. 121. 179, $282 \mathrm{ff}$. Kantstadion 20. 
weiteren Erkenntnisse bilden können. Dass sie das aber körnen, beruht im wesentlichen auf zwei Grundmerkmalen, die ihnen eigen sind, ihrer Voraussetzungslosigkeit und ihrer unmittelbaren Evidenz. Beides hängt aufs engste zusammen. Was absoluter Anfang sein soll, darf weder seinem Inhalt noch seiner Giltigkeit nach von anderem abhängen.

\section{Die Evidenz.}

Betrachten wir zunächst einmal die „Evidenz“. Für die Bestimmung dieses Begriffes wird der Gegensatz zwischen Ding und Erlebnis, zwischen „transzendenter" und ,immanenter" Wahrnehmung massgebend. Jede immanente Wahrnehmung soll notwendig die Existenz ihres Gegenstandes verbürgen. "Richtet sich das reflektierende Erfassen auf mein Erlebnis, so habe ich ein absolutes Selbst erfasst, dessen Dasein prinzipiell nicht negierbar ist, d. h. die Einsicht, dass es nicht sei, ist prinzipiell unmöglich“. „Zu jedem Erlebnisstrom und Ich als solchem gehört die prinzipielle Möglichkeit, diese Evidenz zu gewinnen, jeder trägt die Bürgschaft seines absoluten Daseins als prinzipielle Möglichkeit in sich selbst". ${ }^{1}$ ) Auch wenn ein Ich in seinem Erlebnisstrome nür Phantasien, nur fingierende Anschauungen hätte, so wäre doch das fingierende Bewusstsein nicht selbst ein fingiertes, vielmehr gehört auch hier zu seinem Wesen, wie zu jedem Erlebnis, „die Möglichkeit wahrnehmender und das absolute Dasein erfassender Reflexion".2) Eine genauere Begriffsbestimmung der Evidenz ergibt sich aber noch aus dem Unterschied zwischen dem „assertorischen" Sehen eines Individuellen, z. B. dem „Gewahren" eines Dinges oder eines individuellen Sachverhaltes, und dem „apodiktischen“ Sehen, dem Einsehen eines Wesens oder Wesensverhaltes, die ausserdem, nämlich bei der Anwendung einer Wesenseinsicht auf assertorisch Gesehenes, auch in einer durch die Mischung bedingten Modifikation auftreten können. Evidenz im allgemeinen kommt beidem $\mathrm{zu}$, aber nur dem zweiten „apodiktische Evidenz" ${ }^{3}{ }^{3}$ ) Mit Schärfe wird betont, dass es sich bei der Evidenz nicht etwa um einen „dem Akte irgendwie angehängten Inhalt, um ein Beigefügtes welcher Art immer" handelt,

1) Ideen S. 85.

2) Ideen S. 285.

3) Ideen S. 85. 
sondern um „einen eigentümlichen Setzungsmodus“. Evidenz sei „nicht irgend ein Bewusstseinsindex, der an ein Urteil angeheftet, uns wie eine mystische Stimme aus einer bessern Welt zuruft: Hier ist die Wahrheit!" Andernfalls wären ja die Bedenken zu erwägen, „die keine Index- und Gefühlstheorie der Evidenz überwinden kann: ob nicht ein Lügengeist (der Cartesianischen Fiktion) oder eine fatale Änderung des faktischen Weltverlaufs es bewirken könnte, dass gerade jedes falsche Urteil mit diesem Index, diesem Gefühl der Denknotwendigkeit, des transzendenten Sollens u. dgl. ausgestattet wäre." ${ }^{1}$ )

Knüpfen wir sogleich an diesen letzteren Punkt an, so sehen wir uns zu der Gegenfrage herausgefordert: Ist denn die hier vertretene Evidenztheorie, ist überhaupt irgendeine Theorie in der Lage, skeptische Bedenken dieser Art, wie sie in der Cartesianischen Fiktion des Lügengeistes ihren extremen Ausdruck gefunden haben, zu ïberwinden? Ist denn der "Wesensforscher" besser daran, wenn ein anderer „Wesensforscher" bei einer Exemplifikation durch ein wirkliches oder ein Phantasieerlebnis das Wesen dieses Erlebnisses anders erfasst, als er selbst? Wie will er dem, der die vermeintliche Zuverlässigkeit der „Wesensschauung“ für Selbsttäuschung hält, das Gegenteil beweisen? Er verlangt von ihm, dass er es versuche, die "phänomenologische Einstellung“ zu vollziehen, er betont die Schwierigkeiten und Vorurteile, die besonders der Empirist zu überwinden hat, um die „reinen Gegebenheiten“ zu erfassen und erwartet, dass an ihm, sofern er richtig eingestellt ist, dieselbe unmittelbare Evidenz der "Wesensschauung“ sich erweise. Genau in derselben Lage befindet sich der empirische Forscher, der voraussetzt, dass ein anderer Beobachter durch dasselbe Evidenzbewusstsein, das ihn selbst leitet, auf Grund einer Wahrnehmung und Beobachtung derselben Erlebnisse zu denselben richtigen Urteilen geführt werde, wie er selbst, nur dass er dabei nicht ein bisher unbekanntes "Schauen" von seinem Gegner verlangt, sondern dasjenige Anschaung und Denken verbindende Verfahren, das in der Praxis der Wissenschaft längst bewährt ist. Die Behauptung eines das giltige Urteil begleitenden Evidenzbewusstseins hat ja richtig verstanden nicht den Sinn, dass aus einer inneren Wahrnehmung der Evidenz oder gar in induktiver Ableitung aus den Tatsachen

1) Ideen S. 300 . 
der Evidenz die Giltigkeit eines Urteils erschlossen werden soll. Indem wir von Evidenzbewusstsein ${ }^{1}$ ) reden, stellen wir vielmehr nur auf Grund psychologischer Analyse dasjenige psychische Moment fest, auf welchem der Vollzug richtiger Urteile beruht, genau so, wie Husserl phänomenologisch die „Wesenserschauung“ als den Weg zur Gewinnung richtiger Urteile über Erlebnisse erkennt. Die psychologische Feststellung des in einem bestimmten Fall vorhandenen Evidenzgefühls ist natürlich nicht der Grund, ein Urteil als richtig zu behaupten, sondern das Erlebnis dieser Evidenz als solches, das dem Urteilenden in der Regel überhaupt nicht deutlich zum Bewusstsein kommt. In einem Streitfall steht Evidenzbewusstsein gegen Evidenzbewusstsein, genau so, wie „Wesenserschauung“ gegen „Wesenserschauung" steht.

\section{Reflexion und Selbstbeobachtung und}

die Überwindung des Zweifels an ihren Ergebnissen.

Doch nein! Wir können es weder bei dieser blossen Gegenüberstellung des Für und Wider bewenden lassen, da ja doch eine Entscheidung zwischen wahr und falsch möglich sein muss, noch auch bei der scheinbaren Gleichberechtigung der die Richtigkeit des Urteils begründenden Momente, da eine nähere Betrachtung

1) Welche Qualität diesem Evidenzbewusstsein zukommt, ob es seinem Kerne nach ein Gefühl ist oder etwas anderes, darum handelt es sich hier nicht. Vgl. zu der erkenntnistheoretischen Seite der Frage mein Werk über Fries und Kant II, S. 95 ff. zur psychologischen Seite mein Lehrbuch der Psychologie S. 289 ff., Husserls Bemerkung (Ideen S. $39 \mathrm{ff}$.) : die letztgenannten Darstellungen des Lehrbuchs seien „psychologische Fiktionen ohne das mindeste Fundament in den Phänomenen" habe ìch mit einiger Verwunderung gelesen. Sollten die dort erwähnten Gefühle intellektueller Befriedigung, die Husserl selbst mit der „Freude über einen frei und fruchtbar ablaufenden theoretischen Gedankengang“ (Ideen S. 146) anführt, sollte das vielerörterte "Bekanntheitsgefühl" und die auch von Sigwart vertretenen "Evidenzgefühle" (der Glaube an das Recht dieses Gefühls ist nach Sigwart "der letzte Ankergrund aller Gewissheit überhaupt"), lauter Erscheinungen, deren Vorkommen, wenn wir von dem hier nicht in Betracht kommenđen Streit um die Gefühlsqualităt absehen, von den verschiedensten wisssenschaftlichen Beobachtern bestätigt wird, wirklich in den "Phänomenen" nicht das "mindeste Fundament" haben? Mir scheint: hier ist wirklich das oft missbrauchte Wort: „Wer im Glashause sitzt, usw. schwer zu unterdrücken. Wer uns zumutet, „reine Wesen“, reine "Gegebenheiten" zu erschauen, die ein "absolutes Sein" darstellend weder Begriffe noch Anschaungsinhalte in einem uns bekannten Sinne sind, müsste, glaube ich, mit der Anklage der Fiktion zurückhaltender seín. 
der angeblich „apodiktischen Evidenz" jener "Wesenserschauung“ mit Notwendigkeit über diese selbst hinausführt. Natürlich genügt das blosse Haben eines Erlebnisses nicht; es muss seinem Wesen nach erschaut werden. Aber auch dieses Erschauen reicht noch nicht aus, wenn er diese Erkenntnis andern vermitteln, ja wenn er sie auch nur für sich selbst als eine klare und vollständige Erkenntnis besitzen will. Er muss sie in Begriffe fassen und diese Begriffe mit Worten bezeichnen. Das erschaute Wesen muss dabei zunächst durch die Reflexion hindurch. Über die Schwierigkeit, die darin liegt, hat sich auch Husserl ausgesprochen. Er bringt sie in Zusammenhang mit den in der Identität des Beobachtenden und des Erlebenden liegenden Schwierigkeiten der Selbstbeobachtung. Zwar habe die Phänomenologie keine Daseinsfeststellungen über Erlebnisse zu machen, also auch keine „Erfahrungen" und "Beobachtungen" in dem natürlichen Sinne, in dem eine Tatsachenwissenschaft sich auf dergleichen stützen müsse; aber sie mache doch, „als prinzipielle Bedingung ihrer Möglichkeit, über unreflektierte Erlebnisse Wesensfeststellungen“. Diese verdanke sie aber der Reflexion, näher der „reflektierten Wesensintuition". Es kommen daher die skeptischen Bedenken hinsichtlich der Selbstbeobachtung insofern auch für die Phänomenologie in Betracht, als sich diese Bedenken "von der immanent erfahrenden Reflexion auf jede Reflexion überhaupt erstrecken lassen ". ${ }^{1}$ ) Husserl meint jedoch, auch dieser, wie jeder echte Skeptizismus, zeige sich durch den prinzipiellen Widersinn an, "dass er in seinen Argumentationen implizite, als Bedingungen der Möglichkeit ihrer Geltung, eben das voraussetzt, was er in seinen Thesen leugnet". „Wer auch nur sagt: Ich bezweifle die Erkenntnisbedeutung der Reflexion, behauptet einen Widersinn. Denn über sein $\mathrm{Zweifeln}$ aussagend reflektiert er, und diese Aussage als gültig binstellen setzt voraus, dass die Reflexion den bezweifelten Erkenntniswert wirklich und zweifellos (sc. für die vorliegenden Fälle) habe, dass sie die gegenständliche Beziehung nicht ändere, dass das unreflektierte Erlebnis im Übergang in die Reflexion sein Wesen nicht einbüsse ${ }^{\text {. }}{ }^{2}$ ) $\mathrm{Da}$ ferner in den Argumentationen beständig die Rede sei von der Reflexion als von einer Tatsache und ebenso von unreflektierten Erlebnissen

1) Ideen $8.151 \mathrm{ff}$.

\%) Ideen S. $155 \mathrm{f}$. 
als Tatsachen, so werde ebendamit beständig ein Wissen von unreflektierten Erlebnissen, darunter von unreflektierten Reflexionen vorausgesetzt, während zugleich die Möglichkeit solchen Wissens in Frage gestellt werde. Nicht der leiseste Rechtsgrund bleibe dann für die Gewissheit übrig, dass es überhaupt ein unreflektiertes Erlebnis und eine Reflexion gebe und geben könne. Hier wie überall verliere die Skepsis ihre Kraft „durch Rückgang von den verbalen Argumentationen auf die Wesensintuition, auf die originär gebende Anschauung und ihr ureigenes Recht". ${ }^{1}$ )

Diese scharf durchgeführte Stellungnahme zum Skeptizismus, in dessen Überwindung eines der stärksten Motive der Phänomenologie liegt, sowie der Versuch, auf diesem Wege das Problem der Selbstbeobachtung gewissermassen auszuschalten, ist aber, wie sich sofort zeigen wird, durchaus abhängig von der bereits berührten Frage nach dem Verhältnis des Erlebnisses zar Aussäge über das Erlebnis. Schon die Analyse der Selbstbeobachtung führt mit Notwendigkeit auf diese Frage.

Beobachtung ist ja nicht identisch mit Wahrnehmung und ebensowenig Selbstbeobachtung mit innerer Wahrnehmung. Der Zoologe, der ein Tier beobachtet, nimmt es nicht bloss wahr, wie ein beliebiger Spaziergänger, sondern er richtet seine Aufmerksamkeit auf das ihn interessierende Objekt und - dies ist der Punkt, der uns hier besonders interessiert - mit der sinnlichen Wahrnehmung verbinden sich unmittelbar und von ihr unabtrennbar alle die begrifflichen Vorstellungen, welche der Beobachter von ähnlichen Objekten bereits besitzt und die sich nun „bereit stellen ", um die wissenschaftliche Erfassung des Objektes, in diesem Fall insbesondere seine Klassifikation, zu ermöglichen. Auch das Verhalten des psychologischen Beobachters ist hiervon nicht grundsätzlich verschieden, sofern auch er nicht umhin kann, im Augenblick der Beobachtung die bisher über das Objekt gewonnenen Begriffe in Anwendung zu bringen. ${ }^{2}$ ) Eben hiervon soll sich nun allerdings die phänomenologische Erfassung des Wesens der Erlebnisse in zweierlei Richtung unterscheiden. Einmal soll diese ein reines Schauen sein, für das gerade das Fehlen der logischen Vermittlung charakteristisch und eine der Garantien

) Ideen S. 156.

2) vgl. hierzu mein Lehrbuch der Psychologie S. 36 ff. und meine Schrift uber Selbstbeobachtung und Experiment in der Psychologie 1897. 
seiner Unfehlbarkeit sei. Und dann wird die Wesenserschaunng, die ja auch beliebiger Phantasieerlebnisse zur Exemplifikation sich bedienen kann, gegenüber jeder Feststellung von Tatsächlichem aufs schärfste abgegrenzt. Mit welchem Rechte wird denn aber überhaupt irgend ein Erlebnis mit einem bestimmten Namen bezeichnet, wenn der "Wesensforscher" nicht Begriffe von Erlebnissen bereits besitzt, die es ihm ermöglichen, ein Erlebnis gerade diesem und keinem andern Begriff unterzuordnen und es dementsprechend zu bezeichnen? Das „Haben“ des Erlebnisses als solches bedeutet ja noch nichts; irgend welcher Wert für die Erkenntnis entsteht erst in dem Augenblick, in welchem das Erlebnis benannt werden kann und damit aus der Sphäre des blossen „Habens“, das dem Menschen für viele Erlebnisse mit dem Tier gemeinsam ist, in die Sphäre des Erkennens erhoben wird.

In diese Erwägungen wird mit Notwendigkeit auch jene ganze Beweisführung hineingezogen, durch welche Husserl den Skeptizismus zu überwinden trachtet. Wenn er den Widersinn eines Skeptizismus, der die Möglichkeit bezweifelt, irgend etwas über den Inhalt eines unreflektierten Erlebnisses und über die Leistung der Reflexion zu konstatieren, darin sieht, dass in den Argumentationen des Skeptikers doch immer von der Reflexion als von einer Tatsache und ebenso von unreflektierten Erlebnissen als Tatsachen die Rede ist, das in Frage gestellte Wissen von der Reflexion und von unreflektierten Erlebnissen also doch als möglich vorausgesetzt wird, so ist damit die Schwäche eines solchen Skeptizismus ohne Zweifel richtig getroffen. Daraus folgt aber noch nicht, dass jenes Wissen als "unmittelbares Wissen" ausschliesslich begründet sein könne „durch reflektive gebende Anschauung" im Husserlschen Sinne. Vielmehr führt diese Ansicht mit Notwendigkeit über sich selbst hinaus. Es ist unbestreitbar, dass viele Erlebnisse, z. B. Freude oder Zorn, sich unter dem Einfluss der auf sie gerichteten Reflexion verändern. Wir müssen nun allerdings annehmen, dass es trotzdem möglich ist, ihr Vorhandensein festzustellen und ihr Wesen zu erkennen, wenn wir nicht jenem unhaltbaren Skeptizismus verfallen wollen; und diese Annahme schliesst grundsätzlich allerdings die Voraussetzung ein, dass „das unreflektierte Erlebnis im Übergang in die Reflexion sein Wesen nicht einbüsse ${ }^{\alpha}{ }^{1}$ ) Aber diese Voraus-

1) Ideen S. 155 . 
setzung bezieht sich auf einen unteilbaren Zeitmoment; und selbst für diesen wäre niemals feststellbar, ob das Erlebnis Freude, Zorn, Reflexion oder irgend etwas anderes ist, wenn der Reflektierende nicht von früheren Erlebnissen her und an Begriffen von diesen Erlebnissen ein Kriterium dafür hätte, was Freude, Zorn, Reflexion ist. Wir sehen: die Wesenserschaung als angebliches unmittelbares und absolutes Wissen führt doch immer wieder in das Denken hinüber und lässt sich von ihm, sofern sie überhaupt Erkenntnis sein will, niemals völlig trennen. Überall, wo wir "Gegebenheiten" erforschen wollen, und sei es auch das „Wesen" dieser „Gegebenheiten“, stehen wir auf dem Boden der Eifahrungswissenschaft, die uns Kant als ein untrennbares Zusammen von Anschauung und Denken nachgewiesen hat.

Jene Forderung eines Kriteriums verstärkt sich aber noch, wenn wir berücksichtigen, dass es eine Bewusśtseinstätigkeit, die auf einen unteilbaren Zeitmoment beschränkt wäre, nicht gibt. Auch die Reflexion, die, soweit es dabei auf die wissenschaftliche Erkenntnis des Psychischen ankommt, mit der Selbstbeobachtung zusammenfällt, nimmt, um zur Wirkung zu gelangen, eine gewisse Zeit in Anspruch. Dann aber bleibt immer die Möglichkeit, dass die Qualität des Beobachteten von dem Augenblick, wo die Reflexionstätigkeit einsetzt, bis zu dem Moment, wo sie ihre volle Wirkung äussert, sich bereits etwas geändert haben könnte. Hier hilft nur die Erinnerung und die Vergleichung der erinnerten Momente unter der Leitung eines aus der Erfahrung gewonnenen Begriffes der in Betracht kommenden Erlebnisse. Nehmen wir an, die Qualität eines Erlebnisses wäre a und das vollständige Vorhandensein derselben wäre mit aaa bezeichnet, so könnte die unter dem Einfluss der Reflexion auftretende Veränderung der Qualität des Erlebnisses symbolisch dargestellt werden in der Reihe: aaa, aab, abc, bcd, cde usw. Jeder Zustand kann, wenn er vorüber ist, in der Erinnerung reproduziert werden und unterliegt insofern nicht dem modifizierenden Einfluss der Reflexion; und der erinnerte Moment kann mit anderen erinnerten Momenten und mit dem erlebten Moment verglichen und an der Hand des die Benennung vermittelnden Begriffes z. B. A entschieden werden, dass die Reihe von bcd an überhaupt nicht mehr zu dem gemeinten Erlebnis gehört. Soweit die Reflexion also überhaupt Erkenntnis sein soll, ist sie niemals bloss "gebende Anschauung", sondern 
immer schon Anwendung von Begriffen, ein Ineinander von Anschauung und Denken.

Die Folgerungen für den Evidenzbegriff sind nicht schwer daraus zu ziehen. Man mag das Wort „Evidenz" auch auf die einzelne Anschauung anwenden, oder man mag wie Husserl das „Einsehen eines Wesens oder Wesensverhaltes" so nennen: in jedem Fall gewinnt der Begriff erst dadurch Erkenntniswert, dass er anf ein in einer Aussage formuliertes Urteil Anwendung findet, ob dieses selbst sich nun auf die Anschauung oder auf andere Urteile gründe. Auch die „anschauliche Evidenz" ist also überall, wo sie wirklich Erkenntnis vermitteln soll, stets zugleich „begriffliche Evidenz". $\left.{ }^{1}\right)$ Es geht nicht an, irgend einen anschaulichen Erkenntnisinhalt, der sich auf Gegebenes bezieht, völlig vou der bisher erworbenen, in Begriffen und Urteilen bereits vorliegenden Erkenntnis loszulösen. Wir nögen vielleicht einmal in einem Gegenwartsaugenblick so leben, dass Vergangenheit und Zukunft versinken und das Erlebnis, in das wir uns vertiefen, innerhalb des gesamten „Erlebnisstroms“ vollkommen isoliert erscheint. Für die Erkenntnis wäre diese Isolierung, auch wenn sie im entwickelten Bewusstsein möglich sein sollte, insofern wertlos, als das Wissen von einem solchen Erlebnis überhaupt erst dadurch Erkenntnis wird, dass das Erlebte in einem Urteil zu den bereits vorhandenen Begriffen in Beziehung gesetzt wird. Dem Mystiker und Ekstatiker zerfliesst in der Hingabe an das All-Eine sein eigenes Ichbewusstsein und damit auch jede Anknüpfung an den sonstigen Zusammenhang seines Denkens. Sobald er aber von dem redet, was sein ganzes Sein erfüllte, um andern dieselben Erkenntnisse zu vermitteln, bedient er sich gewisser Begriffe, die das Gesagte in Beziehung bringen zu seinem sonstigen Wissen und - trotz der grundsätzlichen Ablehnung aller menschlichen Verkleinerung, wie sie am schärfsten und kühnsten in Plotins Enneaden vertreten ist - in irgend einer Weise abhängig macht von menschlich-begrifflichen Vorstellungen.

\section{Die Frage des Kriteriums}

und seines Verhältnisses zur Evidenz.

Zur weiteren Durchdringung dieses Problems der Evidenz ist es nötig, das Verhältnis der Begriffe Evidenz und Kriterium

1) Diese Unterscheidung findet sich besonders scharf bei W. Wundt, Paychologismus und Logizismus, Kleine Schriften I, $627 \mathrm{f}$. 
näher ins Auge $\mathrm{zu}$ fassen. Von Evidenz reden wir da, wo die Wahrheit eines Urteils, sei es nun aus der Anschaung oder unabhängig von ihr gewonnen, „unmittelbar einleuchtet". Sie ist natürlich nicht die Wahrheit selbst, - sie fällt auch nicht zusammen mit dem Inhalt des wahren Urteils. Sie ist vielmehr der psychologische Ausdruck für den Wahrheitscharakter der Wahrheit. Die Begriffsbestimmung der Evidenz, die Husserl in den „Logischen Untersuchungen" gibt, ist von dieser Auffassung nicht so weit entfernt, als es auf den ersten Blick scheinen möchte. Da heisst es: "Wahrheit ist eine Idee, deren Einzelfall im evidenten Urteil aktuelles Erlebnis ist." Und eine eigentliche Definition der Evidenz wird gegeben in dem Satz: „das Erlebnis der Zusammenstimmung zwischen der Meinung und dem selbst Gegenwärtigen, das sie meint, zwischen dem aktuellen Sinn der Aussage und dem selbst gegebenen Sachverhalt ist die Evidenz, und die Idee dieser Zusammenstimmung die Wahrheit." ${ }^{1)}$ Sehen wir ab von dem hierin mit enthaltenen platonisch gewendeten Begriff der Wahrheit und von der These, dass die Evidenz des Urteils ausschliesslich in der „originären Gegebenheit", in der unmittelbaren „Wesenserschauung" wurzeln könne - eine These, die wir schon deshalb ablehnen zu müssen glaubten, weil die erst den Urteilsinhalt zur Evidenz erhebende Formulierung des Urteils den Evidenzanspruch mit Notwendigkeit auch auf die darin zum Ausdruck kommenden von der bisherigen Erfahrung abhängigen begrifflichen Beziehungen erstreckt - so stimmt darin mit unserer Auffassung zusammen, dass der Wahrheitscharakter der Wahrheit in einem besonderen Erlebnis erlebt wird. Denn auch für Husserl ist ja doch die Evidenz nicht das Erleben des Wahrheitsinhalts als solchen, sondern ein Vorgang, in welchem eben das "Wahrheitsein" dieses Inhalts erlebt wird. Welche Qualität man diesem Erlebnis zuschreibt, ob es als ein Gefühl angesehen wird oder als irgend etwas anderes, ist hier unwesentlich. Dagegen ist die Frage natürlich die durchgreifende, in welchem Sinne diese Evidenz als Kriterium zu betrachten ist. Gehen wir von der ursprünglichen Bedeutung von

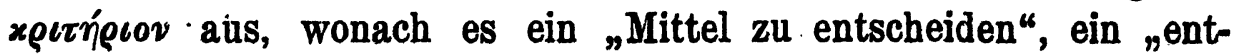
scheidendes Kennzeichen" ist, so gelangen wir zunächst zu der Auffassung, dass das Kriterium ein Mittel ist, zwischen Wahrbeit

1) Husserl, Logische Untersuchungen I, 2. A. S. 190 f. vgl. hierzu auch mein Buch über Fries und Kant II, $96 \mathrm{ff}$. 
und Unwahrheit zu entscheiden. Dies gilt zunächst für das urteilende Subjekt selbst. Wir können diese subjektive Seite des Kriteriums auch kurz als das „subjektive Kriterium“ bezeichnen. Dieses „subjektive Kriterium“ fällt nun zusammen mit der Evidenz. Seine Wirksamkeit besteht aber, wie bereits betont wurde, natürlich nicht darin, dass der Urteilende das Evidenzerlebnis bei sich selbst konstatiert und daraus den Schluss zieht, dass das von ihm begleitete Urteil wahr ist; sondern das Evidenzbewusstsein wirkt für ihn nur als tatsächliches Motiv mit, das Urteil zu vollziehen. Es hat daher auch als Erlebnis des Einen keine Bedeutung für die Zustimmung der andern $z u$ demselben Urteil. Wenn diese zustimmen, so tun sie es natürlich wiederum nicht etwa, weil sie aus dem irgendwie vermuteten oder konstatierten Evidenzbewusstsein der andern den Schluss gezogen hätten, dass deren Urteile wahr seien, sondern weil sie auf grund des gesetzmässig - nicht „zufällig“, wie Husserl will - eintretenden Evidenzbewusstseins, das wir psychologisch allerdings am besten als Gefühl zu fassen glauben, gar nicht anders können, als ihre Zustimmung zu erteilen. Es hat also auch keinen Sinn; sich andern gegenüber auf dieses Evidenzgefühl zu berufen; denn entweder ist es da oder es ist nicht da. Wir können andere Urteilende, die wir überzeugen wollen - und darum handelt es sich natürlich - nur veranlassen, die Anschaungs- und die Denkakte zu vollziehen, bei denen dieses Evidenzgefühl sich einstellt. Die Richtigkeit des Vollzugs dieser Akte ist das nobjektive Kriterium" der Wahrheit, das einzige, auf das wir uns berufen können, wenn es sich darum handelt, in der Auseinandersetzung mit anderen gemeinsam die Wahrheit zu erforschen. Auch, wo die Bedingungen menschlichen Erkennens selbst zum Gegenstand der Forschung gemacht werden, wie dies z. B. in Kants Kritik der reinen Vernunft der Fall ist, verhält es sich nicht wesentlich anders. Von jenem Ineinander von Anschanung und Denken aus, das wir "Erfahrung" nennen, müssen in richtig vollzogenem Regressus die Bedingungen der Möglichkeit dieser Erfahrung abgeleitet werden, und als objektives Kriterium erscheint hier das Prinzip der "Möglichkeit der Erfahrung“, Da diese Forschungsarbeit aber, sofern sie selbst Erkenntnis ist, die Möglichkeit des Erkennens schon voraussetzt, kann sie diese Möglichkeit nicht selbst erst beweisen, sondern sie muss sich darauf verlassen, dass jenes letzte, subjektive Kriterium, das den Hintergrund aller An- 
erkennung von Wahrheiten bildet, seine Schuldigkeit tut. Das objektive Kriterium aber ist auch hier unentbehrlich, da auf ihm allein die Möglichkeit beruht, für die Entscheidung zwischen Wahrheit und Unwahrheit einen gemeinsamen Boden $z \mathfrak{u}$ finden.

Aber gerade hier ist der Punkt, wo das Prinzip der Wesensschauung kaum eine befriedigende Lösung als möglich erscheinen lässt. Das gemeinsame Suchen nach der Wahrheit ist von der Möglichkeit abhängig, andere von der Richtigkeit des eigenen Ergebnisses zu überzeugen. Dies aber setzt wiederum die Möglichkeit voraus, den andern dazu zu bringen, dass er gewissen gemeinsamen für beide giltigen Kriterien der Wahrheit sich unterwerfe. $\mathrm{Da}$ eine äussere Nötigung ausgeschlossen ist, kann es sich nur um eine innere psychische Nötigung handeln, die als solche zunächst subjektiver Art, die aber an objektive von jedem Denkenden nacherlebbare Momente geknüpft ist.

Für die Phänomenologie gibt es im Grunde solche objektive Kriterien überhaupt nicht. ${ }^{1}$ ) Sie fordert von demjenigen, der das Wesen der in Betracht kommenden Objekte erkennen will, die "phänomenologische Einstellung", und wer diese phänomenologische Einstellung vollzieht, „erfasst" oder „erschant ohne weiteres das Wesen jener Objekte, der Erlebnisse. Derjenige, der eine solche Wesensschauung nicht vollziehen zu können behauptet, wird auf die Schwierigkeit des Verfahrens, auf die Notwendigkeit der Übung und auf die Voraussetzung einer völligen Loslösung von allen Vorurteilen des gewöhnlichen empirischen Verfahrens hingewiesen. Ein solches Erkenntnisverfahren stellt sich aber jenseits aller sonstigen Kriterien der Erkenntnis. Jedem Einwand gegen ein Ergebnis solcher Wesensforschung, welcher erfahrungsmässig bekannten Tatsachen entnommen ist, ist entgegenzuhalten, dass er nicht der richtigen Methode entstammt. Denn sobald einmal die Möglichkeit einer Korrektur der Wesensforschung durch die Tatsachenforschung zugegeben wäre, würde der ersteren ihr Sonderrecht und ihre grundlegende Bedeutung genommen. Eine solche Position scheint unangreifbar und ist doch zugleich - wenigstens erkenntnistheoretisch betrachtet - hilflos gegenüber jedem Angriff. Denn nachdem sie einen besonderen, auf die bisher erprobte Weise nicht kontrollierbaren, nur dem besonders sich „einstellenden" gangbaren.Weg der

1) vgl. hierzu R.Hønigswald, Prinzipien der Denkpsychologie, 1913, S.30. 
Erkenntnis behauptet, ermöglicht sie auch dem Gegner, seinerseits. ein besonderes der bisher erprobten Kontrolle der Wissenschaft nicht unterworfenes Verfahren, die Wahrheit zu ermitteln, für sich in Anspruch zu nehmen. Aber auch die einer solchen esoterischen Lehre zustimmenden Forscher selbst haben kaum eine Möglichkeit, sich unter sich kritisch auseinanderzusetzen. Zum mindesten wird sich der kritische Ausgleich der auf gemeinsamem Boden erwachsenden Forschungen nur auf Sekundäres beziehen. Denn die. Wesensschauung als solche ist ja absolut, sie unterliegt keiner Korrektur durch induktive Ableitung aus Gegebenheiten. Behauptung steht gegen Behauptung; der eine erschaut dies als. Wesen eines Erlebnisses, der andere jenes.

Alle diese Schwierigkeiten fallen weg, wenn wir Husserls. Phänomenologie ihres scholastisch-apriorischen Gewandes entkleiden und in ihr den energischen und mit Scharfsinn durchgeführten Versuch sehen, einer modernen deskriptiven Psycho$\operatorname{logie}^{1}$ ) zuverlässige begriffliche Grundlagen und ein von der Vermischung mit naturwissenschaftlichen Methoden freies Verfahren zu sichern. Die bisherige Arbeit der Phänomenologie und ihr geschichtliches Recht lässt sich, wie wir bereits gesehen haben, ohne Schwierigkeit unter diesem Gesichtspunkt betrachten. Ihrer völligen Identifikation mit Psychologie steht aber noch ein letzter grundsätzlich wichtiger Gesichtspunkt entgegen, der gesonderte Betrachtung erfordert.

\section{Die Voraussetzungslosigkeit der Phänomenologie in ihrem Verhältnis zur Erkenntnistheorie.}

Die Psychologie ist nach Husserl eine Erfahrungswissenschaft, die als solche eine philosophische Bearbeitung ihres Erfahrungsgebietes, nämlich eine „systematische, das Psychische immanent erforschende Bewusstseinswissenschaft", bereits voraussetzt. Diese Wissenschaft ist eben die Phänomenologie. Sie ist die voraussetzungslose Grundlage aller Philosophie überhaupt. Fordert man von einer wissenschaftlichen Philosophie eine erkenntnistheoretische Begründung, und wiederum von einer erkenntnistbeoretischen Untersuchung, die ernstlichen Anspruch auf Wissenschaftlichkeit erhebt, dass sie dem Prinzip der Voraussetzungslosigkeit genüge,

1) vgl. hierzu auch H. Maier, Logik und Psychologie, Festschrift für Riehl (1914) S. $360 \mathrm{ff}$. 
so kann dieses Prinzip nach Husserl nicht mehr besagen wollen, nals den strengen Ausschluss aller Aussagen, die nicht phänomenologisch voll und ganz realisiert werden können ". ${ }^{1)}$ Die Phänomenologie kann ja dieser Aufgabe genügen, da sie, ohne irgendwelche des Beweises bedürftige Annahmen vorausschicken zu müssen, vorurteilslos nur "reine Gegebenheiten" beschreibt. Sie liefert damit absolute Anfänge und zuverlässige deskriptive Grundlagen aller Erkenntnis und ermöglicht es dadurch, die Philosophie aus dem Stadium der Unwissenschaftlichkeit in das der "strengen Wissenschaft" überzuführen. ${ }^{2}$ )

Wir berühren damit das vielleicht stärkste Denkmotiv der ganzen Phänomenologie. Husserl ist sich bewusst, wie nahe er dem Versuch Descartes' kommt, den universellen $Z$ weifel durch die Reflexion auf ein unmittelbar Gewisses, in Bewusstsein Gegebenes zu überwinden. . Es erscheint ja zunächst nur als eine modernere Fassung jenes Cartesianischen Grundgedankens, wenn der Möglichkeit, dass ein Ich in seinem Erlebnisstrom „nur Phantasien", nur "fingierende Anschauungen" hätte, der Satz gegenübergestellt wird: "Das Vorschwebende mag ein blosses Fiktum sein, das Vorschweben selbst, das fingierende Bewusstsein ist nicht selbst fingiertes, und zu seinem Wesen gehört, wie zu jedem Erlebnis, die Möglichkeit wahrnehmender und das absolute Dasein erfassender Reflexion ${ }^{*} .^{3}$ ) Ein Doppeltes charakterisiert aber den wesentlichen Unterschied. An die Stelle des Cartesianischen Zweifels tritt die universelle "Ausschaltung" oder "Einklammerung" der gesammten Erfahrungswelt, die nur die Welt des "reinen Bewusstseins", die "Welt als Eidoș" übrig lässt. Zweitens aber wird das Kriterium dẹr von diesem Ausgangspunkt aus fortschreitenden Erkenntnis nicht rationalistisch in der Klarheit und Deutlichkeit des Denkens, sondern intuitionistisch in der "Wesensschauung" gefunden. Das zweite dieser Grundmerkmale hat uns bereits eingehend beschäftigt, das erste aber ist durchaus massgebend für die Art der Voraussetzungslosigkeit der Phänomenologie in ihrem Verhältnis zur Erkenntnistheorie.

Dieser wichtige Punkt erhält neues Licht, wenn wir dem Standpunkt der Phänomenologie einen anderen modernen philosophischen

1) Log. Unt. II, 19.

2) E. Husserl, Philosophie als strenge Wissenschaft, Logos $289 \mathrm{ff}$.

э) Ideen S. 85. 
Versuch gegenüberstellen, von einem gegebenen Ausgangspunkt möglichst voraussetzungslos die Grundlagen der Philosophie zu entwickeln. Nach dem von R. Avenarius begründeten Empiriokritizismus ist - ähnlich wie bei Husserl die "natürliche Einstellung“ - der „natürliche Weltbegriff" der "natürliche Ausgangspunkt alles Philosophierens". Die Weltbegriffe der Philosophie sind nur Variationserscheinungen desselben. Dieser natürliche Weltbegriff zerfällt aber, unter einem formalen Gesichtspunkt betrachtet, alsbald in zwei logisch verschiedenwertige Bestandteile: eine „Mannigfaltigkeit von tatsächlich Vorgefundenem" und eine "Hypothese“. Der erstere, der "empiriokritische Befund", scheidet sich wieder in zwei Hauptteile, das "Ich" und die "Umgebung", deren gegenseitige Beziehung unauflöslich ist und daher "empiriokritische Prinzipalkoordination" genannt wird. Der zweite Bestandteil des natürlichen Weltbegriffs besteht darin, „dass ich den mitmenschlichen Bewegungen, welchen, sofern sie nur als ein von meinem örtlichen Standpunkte aus Vorgefundenes betrachtet werden, tatsächlich nur eine mechanische Bedeutung zukommt, eine mehr als mechanische Bedeutung zuschreibe"..1) Indem nun aber die herrschende Psychologie dieses noch anzunehmende "Amechanische" als "Empfindungen", die ihren Ort im "Gehirn" haben, in uns hineinverlegt, wird durch diese "Introjektion" der ganze natürliche Weltbegriff gefälscht, und es entsteht erst dadurch die ihm fremde Scheidung in eine äussere und innere Welt. Die „Kritik der reinen Erfahrung" soll dann diese Introjektion wieder ausschalten, um den unvariierten natürlichen Weltbegriff wieder herzustellen. $\left.{ }^{2}\right)$ Der Weg aber, auf dem dies geschieht, zeigt, dass schon das "Vorgefundene" im Lichte einer bestimmten Wissenschaft betrachtet wurde. ${ }^{1}$ ) Die menschlichen Individuen erscheinen als "hochentwickelte Organismen" mit einer "Mehrheit von Teilsystemen", die „Umgebungsbestandteile“ als \#̈nderungsbedingungen für den Organismus, das dem Gehirn entsprechende zentrale nervöse Teilsystem $C$ tritt völlig an die Stelle dessen, was dem natürlichen Erkennen etwa als "Ich" oder als Selbstbewusstsein gilt, und das

2) vgl. R. A venarius, der menschliche Weltbegriff, Leipzig 1891 S. $144 \mathrm{ff}$. und „Bemerkungen zum Begriff des Gegenstandes der Psychologie“ Vierteljahrsschrift für wissenschaftl. Philosophie 1894. 174. 153.

2) R. Avenarius, Kritik der reinen Erfahrung 1888, I, VII. 
ganze System steht durchaus unter dem Gesichtspunkt der Erhaltung des Gesamtorganismus. ${ }^{1}$ )

Was also hier herauskomnt, ist nicht eine Variationserscheinung des natürlichen Weltbegriffs, sondern eine Beseitigung desselben zugunsten eines andersartigen wissenschaftlichen Weltbegriffs, nämlich des biologischen, der bereits die Art und Weise der Beschreibung 'des Vorgefundenen bestimmt hat.

\section{Der Begriff der Intention und die Bildertheorie.}

Formal dieselbe, wenn auch material eine ganz anders orientierte Vermischung eines natürlichen und eines wissenschaftlichen Weltbegriffs scheint mir nun die Phänomenologie Husserls za enthalten. "Ich und Umgebung“, der "empiriokritischen Prinzipalkordination", entspricht bei Husserl die "Intentionalität". Dieser Begriff schliesst sich an Franz Brentanos Abgrenzung der "psychischen Phänomene" an, der jedes psychische Phänomen "durch das charakterisiert" sein lässt, "was die Scholastiker des Mittelalters die intentionale (auch wohl mentale) Inexistenz eines Gegenstandes genannt haben, und was wir, obwohl mit uicht ganz unzweideutigen Ausdrücken, die Beziehung auf einen Inhalt, die Richtung auf ein Objekt (worunter hier nicht eine Realität zu verstehen ist) oder die immanente Gegenständlichkeit nènnen würden". Auch für Husserl ist das Bewusstsein eine zusammenfassende Bezeichnung für ,jederlei, "psychische Akte" " oder "nintentionale Erlebnisse" ". Es handelt sich dabei aber nicht, wie Brentayos Ausdrucksweise nahelegen könnte, um einen realen Vorgang oder ein reales sich Beziehen, das sich zwischen dem Bewusstsein oder Ich und der "bewussten" Sache abspielen würde, auch nicht um ein Verhältnis zwischen zwei gleicherweise im Bewusstsein real zu findenden Sáchen: ${ }_{n}$ Akt und intentionales Objekt". Im intentionalen Erlebnis ist ein Gegenstand "gemeint", es ist auf inn "abgezielt" "und zwar in der Weise der Vorstellung oder zugleich der Beurteilung usw." und darin liegt nichts anderes, als „dass eben gewisse Erlebnisse präsent sind, welche einen Charakter der Intention haben und speziell der vorstellenden,

1) Aqvenarius, Kritik der reinen Erfahrung I, 32 ff. Von der Frage, inwieweit schon in dem Vorgefundenen psychologische Voraussetzungen mitenthalten sind, die, wie mir scheint, zu bejahen ist, (vgl. mein Buch Fries und Kant I, $15 \mathrm{ff}$.) soll hier abgesehen werden. 
urteilenden, begehrenden Intention usw. "1) Ein solches Erlebnis kann natürlich mit dieser seiner Intention im Bewusstsein vorhanden sein, ohne dass der Gegenstand überhaupt existiert und vielleicht gar existieren kann. „Jupiter stelle ich mir nicht anders vor als Bismarck, den Babylonischen Turm nicht anders als den Kölner Dom, ein regelmässiges Tausendeck nicht anders als einen regelmüssigen Tausendflächner ${ }^{\text {(2) }}{ }^{2}$ ) Damit tritt ein grundwesentlicher Unterschied hervor zwischen Sein als Erlebnis und Sein als Ding. Zum Wesen des Erlebnisses gehört es, dass es in immanenter Wahrnehmung wahrnehmbar ist, zum Wesen eines Raumdinglichen aber, dass es das nicht ist. ${ }^{8}$ ) Wir bezeichnen daher das Ding als "schlechthin transzendent". $\mathrm{Zu}$ dieser prinzipiellen Unterschiedenheit der Seinsweisen, der kardinalsten, die es überhaupt gibt, zwischen Bewusstsein und Realität, zwischen Immanenz und Transzendenz, gehört aber auch ein "prinzipieller Unterschied der Gegebenheitsart". Ein Ding nehmen wir dadurch war, dass es "sich abschattet" nach seinen verschiedenen Bestimmtheiten. Ein Erlebnis, ein Gefühlserlebnis z. B. schattet sich nicht ab. "Blicke ich darauf hin, so habe ich ein Absolutes, es hat keine Seiten, die sich bald so bald so darstellen könnten. " ${ }^{4}$ )

Die erkenntnistheoretische Bedeutung der Intentionalität wird noch deutlicher nach der negativen Seite in der Ablehnung jeglicher „Bilder-" oder "Zeichentheorie". Wenn man sagt: "Draussen" ist das Ding selbst, im Bewusstsein ist als sein Stellvertreter ein Bild, so übersieht man völlig den wichtigsten Punkt, nämlich, „dass wir im bildlichen Vorstellen auf Grund des erscheinenden "\#ildobjekts" ${ }^{4}$ das abgebildete Objekt (das ${ }_{n}$ Bildsujet" ") meinen." ) Die Bildertheorie vermag ja nicht zu erklären, wie „wir über das im Bewusstsein allein gegebene „„Bild“ “ hinanskommen und es als Bild auf ein gewisses bewusstseinsfremdes Objekt zu beziehen vermögen ". Auch die gegenseitige Ähnlichkeit, die zwischen Bild und Sache behauptet wird, macht ja das eine nicht zum Bilde des andern. Das Bewasstsein selbst müsste dem wahrnehmungsmässig ihm erscheinenden Objekt erst die "Geltung“ oder „Bedeutung“ eines Bildes verleihen. Die Auf-

1) Log. Unt. II, $366 \mathrm{ff}$.

7) Log. Unters. I, 373. vgl. Ideen S. $64 f$.

э) Ideen S. $76 \mathrm{ff}$.

4) Ideen S. $81.76 \mathrm{ff}$.

9) Log. Unt. II, 422 ff. Ideen S. 78 ff. 99.186.

Kantutudion $x$. 
fassung als Bild setrit also selbst schon ein dem Bewusstsein intentional gegebenes Objekt voraus, und würde offenbar auf einen unendlichen Regress führen, da das Objekt selbst immer wieder durch ein Bild konstitaiert sein müsste. Auch die Zeichentheorie unterliegt demselben Einwand. Denn auch sie setzt ein „fundiertes Aktbewusstsein" voraus, damit das Zeichen auf den Gegenstand bezogen werde. Es ist überhaupt ein schwerer Irrtum, wenn man "einen reellen Unterschied zwischen den " "bloss immanenten ${ }^{4 *}$ oder " "intentionalen“" Gegenständen auf der einen und ihnen ev. entsprechenden ${ }_{n}$, wirklichen" " und " "transzendenten " "Gegenständen auf der anderen Seite macht". Man braucht es vielmehr nur auszusprechen, und jedermann muss es anerkennen: dass „der intentionale Gegenstand der Vorstellung derselbe ist wie ihr wirklicher und gegebenenfalls ihr äusserer Gegenstand, und dass es widersinnig ist, zwischen beiden $\mathrm{zu}$ unterscheiden. Der transzendente Gegenstand wäre gar nicht Gegenstand dieser Vorstellung, wenn er nicht ihr intentionaler Gegenstand wäre." $)$

Damit ist die erkenntnistheoretische Stellung oder - wie wir im Sinne der Phänomenologie besser sagen würden - die nicht-erkenntnistheoretische oder vor-erkenutnistheoretische Stellung der Phänomenologie mit aller wünschenswerten Schärfe gekennzeichnet.

Knüpfen wir zunächst an die Polemik gegen die Bildertheorie an, so läge es nahe, zuvörderst daran zu erinnern, dass die Ansicht, unsere Wahrnehmung könne an die Dinge nur herankommen durch blosse "Abschattungen" derselben, während Erlebnisse sich nicht "abschatten“, doch mit der Bildertheorie recht viel Ähnlichkeit hat, sofern der Schatten als ein auf die Umrisslinien reduziertes Bild des „Abgeschatteten" betrachtet werden kann. Unser Interesse ist jedoch in erster Linie der tieferen Frage zugewandt, inwieweit es der Phänomenologie hier gelungen ist, falschen, in das Verhältnis von Ding und Wahrnehmung hineingetragenen Voraussetzungen eine voraussetzungslose Erfassung der "Gegebenheiten" gegenüberzustellen; und diese Frage wiederum hängt aufs engste zusammen mit der andern nach dem Verhältnis der naiven und der wissenschaftlichen Betrachtungsweise, des "natürlichen" und des "wissenschaftlichen Weltbegriffs".. Es zeigt sich nämlich, dass die hier vorgebrachten Einwände gegen eine richtig ver-

1) Log. Unters. II, 424 f. 
standene Bilder- oder Zeichentheorie sich nur dann als triftig erweisen, wenn beide Betrachtungsweisen mit einander vermischt werden, dass sie aber bei reinlicher Scheidung derselben sich auflösen.

Greifen wir irgend ein Beispiel heraus. "Vor mir liegt in Halbdunkel dieses weisse Papier. Ich sehe, ich betaste es. Dieses wahrnehmende Sehen und Betasten des Papieres .... ist eine cogitatio, ein Bewusstseinserlebnis. Das Papier selbst mit seinen objektiven Beschaffenheiten, seiner objektiven Lage zu dem Raumdinge, das mein Leib heisst, ist nicht cogitatio, sondern cogitatum, nicht Wahrnehmungserlebnis, sondern Wahrgenommenes. Nun kann ein Wahrgenommenes selbst sehr wohl Bewusstseinserlebnis sein; aber es ist evident, dass so etwas wie ein materielles Ding, z. B. dieses im Wahnehmungserlebnis gegebene Papier, prinzipiell kein Erlebnis ist, sondern ein Sein von total verschiedener Seinsart.“ ${ }^{1}$ ) Dass dieses von mir wahrgenommene Papier als "materielles Ding" "ein Sein von total verschiedener Seinsart", eben so wie ich es wahrnehme, ein transzendentes Ding sei, kann ich doch nur behaupten, wenn ich mich in den Standpunkt des naiven Denkens hineinversetze. Tue ich das aber, so muss ich es auch mit voller Konsequenz tun. Für das naive Denken existiert dieses Ding „ausserhalb“ des Wahrnehmenden. Von einem "Bild ${ }^{\text {“ ist }}$ erst die Rede, wenn das Ding nicht mehr wahrgenommen, nicht mehr gesehen, gehört, betastet wird. Er kann sich davon ein Bild machen, das dem wahrgenommenen Ding ähnlich ist. Diese Ähnlichkeit stellt aber nicht Bild und Sache auf die gleiche Stufe, so dass sie vertauschbar wären; denn das Bild ist ja in ihm. Kommt er in den Fall, das Bild mit dem Ding selbst zu vergleichen, so ist die Auswahl dessen, was er "meint", eben durch die Ähnlichkeit des "Inhalts“, der für ihn mit dem „Gegenstand zusammenfällt, gegeben, das Ding selbst aber dadurch hinreichend charakterisiert, dass es "draussen" ist. Auch ein unendlicher Regressus braucht nicht zu entstehen; denn das Bild ist ja doch nur deshalb nötig, weil der Gegenstand selbst nicht da ist; das Bild selbst bedarf dieser Vermittlung nicht.

Gehen wir nun von hier zur wissenschaftlichen Betrachtungsweise über, so sehen wir dabei ab von den ältesten Bildertheorien, von der Erklärung der sinnlichen Wahrnehmung durch Empedokles

1) Ideen S. $61 \mathrm{f}$. 
and Demokrit aus den in die Sinnesorgane eindringenden, von den Dingen sich ablösenden Bilderchen. In einer Kants Philosophie geschichtlich voraussetzenden Erörterung können wir von dem Verhältnis zwischen Ding und Währnehmung nicht reden, ohne uns der Abhängigkeit des „Dinges" von uns, den Wahrnehmenden, von unseren Anschauungs- und Denkformen bewusst zu sein. Der Gegenstand der Erkenntnis wird mit Notwendigkeit zum Problem. Dem entgeht auch der Gegenstand nicht, den wir „meinen". Reden wir einmal von ,intentionalen Erlebnissen" und sind alle Erlebnisse als etwas „Bewusstes" erkannt, so stehen wir bereits auf dem Boden des Kantischen Grundgedankens, und wir können von der in jenen Erlebnissen enthaltenen Gegenstandsbeziehung nicht mehr so sprechen, als ob der damit, ,gemeinte“ Gegenstand unabhängig von unserem Vorstellen und Denken gegeben wäre. Auch die „Wesensschauung“ kann sich dem nicht entziehtn, auch ihr muss der Gegenstand zum Problem werden, nachdem er einmal im intentionalen Erlebnis als miterlebt angesehen und damit in den subjektivierenden Prozess hineingezogen ist. Um eine blosse Beschreibung von Gegebenem kann es sich dann nicht mehr handeln; denn das "Gegebene“, das "Objekt" der Wahrnehmung ist seinem Inhalt nach ja bereits als Geist von unserem Geiste erkannt. An die Stelle der blossen Deskription tritt, wie Natorp in einer Auseinandersetzung mit Husserl sagt, ${ }^{1}$ ) die „Rekonstruktion“. Die Gegenstandsbeziehung von dieser Konsequenz loszulösen, wäre nur dann möglich, wenn wir auf dem Standpunkt der naiven Betrachtungsweise stehen blieben, für die Inhalt und Gegenstand der Wahrnehmung samt allen Empfindungsdaten „ausserhalb“ unseres Bewusstseins gegeben sind. Damit aber fiele die ganze „Erlebnis"-lehre dahin.

Kehren wir ferner nochmals zu der Bilder- und zu der Zeichentheorie (von welchen die letztere nur für die wissenschaftliche Betrachtungsweise in Betracht kommt) zurück, so zeigt sich, dass auch gegenüber einer konsequent durchgeführten wissenschaftlich-erkenntnistheoretischen Betrachtungsweise wenigstens die hier angeführten Einwände sich nicht als stichhaltig erweisen. Stellen wir uns z. B. auf den Kantischen Standpunkt und nehmen an, die unmittelbare Beziehung einer Erkenntnis auf Gegenstände in der Anschauung sei nur dadurch möglich, dass der "Gegenstand

1) P. Natorp, Allgemeine Psychologie I (1912) S. $286 \mathrm{ff} .33 \mathrm{ff}$. 
das Gemüt auf gewisse Weise affiziere", so fallen im Augenblick der Anschauung "Bild" oder "Zeichen" des Gegenstandes mit diesem selbst zusammen; in der Reproduktion aber liegt die Beziehung auf den Gegenstand allerdings nur in der Ähnlichkeit des "Bildes" mit diesem selbst oder in der Vertretung desselben durch das "Zeichen", was sich auch darin zeigt, dass das "Bild" oder „Zeichen" irrtümlich auch auf einen andern ähnlichen Gegenstand bezogen werden kann. Ein unendlicher Regressus ist nicht die notwendige F'olge, da die Vertretung des Gegenstandes durch ein Bild oder Zeichen durch die Abwesenheit einer äusseren „Affektion" bedingt ist, eine Vertretung der Vertretung aber unnötig erscheint. Um die sonstigen erkenntnistheoretischen Schwierigkeiten, die zweifellos einer solchen Vorstellungsweise anhaften, handelt es sich hier nicht. Es sollte nur gezeigt werden, dass eine kritische Auseinandersetzung mit der Bildertheorie nicht mit Notwendigkeit auf die Lehre von der Intention führt, dass vielmelr die sich hierbei herausstellenden Schwierigkeiten aus einer Vermischung des naiven und des wissenschaftlich-erkenntnistheoretischen Standpunktes entstehen, indem einerseits der „Gegen-

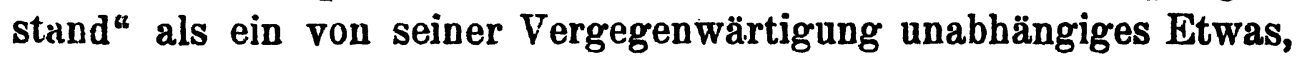
andererseits die Wahrnehmung des "Gegenstandes" im Ganzen als ein Bewusstseinserlebnis betrachtet wird.

Dadurch wird aber zugleich die Voraussetzungslosigkeit der Phänomenologie an einem wichtigen Punkte eingeschränkt. Das „absolute Sein des Immanenten“, das als solches die unmittelbare "Wesensschauung“ als Grundlage aller Wissenschaft ermöglicht, entlehnt seine Absolutheit dem Gegensatz gegen das "bloss phänomenale Sein des Transzendenten “. Das letztere ist aber weder als "Transzendentes“, als „Ding“ im naiven, noch als "Transzendentes“. im erkenntnistheoretischen Sinn "bloss phänomenal“. Es erhält diesen Charakter erst, indem in die voraussetzungslose Betrachtung der "Gegebenheiten" beim Übergang von der "natürlichen" zur "phänomenologischen Einstellung" ein ganz bestimmter Begriff des "Erlebnisses“ hereinkommt. Wir sehen also: wie in dem andern hervorragenden Versuch, vorurteilslos vom „Vorgefundenen" auszugehen, in demjenigen von Avenarius in die Beschreibung sich mehr und mehr, eine - man möchte sagen biologisierende Betrachtungsweise mischt, so ist es hier eine - erkenntnistheoretisch gewiss mögliche, aber mit dem „natür- 
lichen Weltbegriff" nicht übereinstimmende - psychologisierende ${ }^{1}$ ) Betrachtungsweise, welche die Beschreibung des unmittelbar Gegebenen fast unnerklich in einer bestimmten Richtung modifiziert.

VII. Die Notwendigkeit eines im "praktischen Realismus“ gegebenen voraussetzungslosen Ausgangspunktes.

Aber auch hier, wie in dem früher besprochenen Eintreten der Phänomenologie für eine der Naturwissenschaft gegenüber selbständige deskriptive Psychologie, liegt ein Denkmotiv zu Grunde, dessen Befriedigung in einer so geschlossenen logischsystematischen Form an sich schon der Phänomenologie ihre Bedeutung und ihr geschichtliches Recht sichert. Beschäftigt sich die Erkenntnistheorie mit den im Erkennen als solchem liegenden Voraussetzungen alles Wissens, so ist es eine um so wichtigere Frage, inwiefern sie selbst voraussetzungslos sein oder auf voraussetzungslose Anfänge sich gründen kann. In keinem Falle wird sie dabei umhị können, irgendwie von "Gegebenheiten", zum mindesten von dem Erkennen, ihrem Gegenstand, als „Gegebenheit" auszugehen.

Dies tritt selbst in einer Erkenntnistheorie, die jeden empirischen Einschlag in der Begründung so sorgfältig $\mathrm{zu}$ meiden sucht, wie die Kantische, deutlich hervor. Sucht Kant die Erkenntnisprinzipien als Bedingungen der Möglichkeit der Erfahrung oder - was hier dasselbe bedeutet - der Erfahrungserkenntnis nachzuweisen, so ist diese Beweisführung nur dann triftig, wenn Erfahrung möglich sein muss, nämlich, weil sie wirklich ist. Die Erfahrung als eine „Urtatsache“, wie Kuno Fischer sagt,") bildet daher den Ausgangspunkt der Kantischen Erkenntnistheorie, und zwar „Erfahrung“ nicht etwa bloss im empirischen Sinne des „Rohstoffs sinnlicher Eindrücke“, sondern in dem prägnanten Sinne der bereits erfolgten Verarbeitung dieses Rohstoffs durch die Verstandestätigkeit, da ja ihr Vorhandensein in diesem Sinne von der transzendentalen Deduktion der Kategorien vorausgesetzt wird. Da aber diese Erfahrung doch auch nicht, ohne einen unerträglichen Zirkel hervorzurufen, die philosophisch bearbeitete und

1) im weitesten auch die Phänomenologie mit umspannendem Sinne.

ๆ) K. Fischer, Kritik der Kantischen Philosophie S. 91, 99 ff. Ähnlich, wenn auch von anderem Standpunkt aus A. Riehl, Philos. Kritizismus I, 303: "der Begriff der Erfahrung ist der feste Grund, die einzige Voraussetzung der Kantischen Erkenntnistheorie.“ 
in ihre Bestandteile bereits gesonderte Erfahrung des Erkenntnistheoretikers sein kann, so muss es, als Ausgangspunkt wenigstens, die vorwissenschaftliche, oder besser vor-erkenntnistheoretische, die "gemeine" Erfahrung sein, und es vollzieht also auch die theoretische Vernunft, prinzipiell betrachtet, wie die „praktische" bei Kant etwas wie einen „Übergang" von der "gemeinen" „Vernunfterkenntnis" zur ,philosophischen". ${ }^{1}$ )

Wir haben diesen Gedankengang, insbesondere die Frage, wie sich dazu der apriorische Charakter der Erkenntnisprinzipien verhält, hier nicht weiter zu verfolgen. ${ }^{2}$ ) Wir stellen nur fest, dass auch bei modernen Forschern, auch bei solchen, bei denen sich keinerlei Zugeständnisse an den Empirismus vermuten lassen, sich die Unvermeidlichkeit eines solchen Ausgangspunktes verfolgen lässt. Als Beispiel sei Rickert angeführt, der die Notwendigkeit eines von der Erkenntnistheorie vorausgesetzten Objektes, nämlich eben des Erkennens selbst, stark betont. ${ }^{3}$ ) Ob dieses Erkennen las Erkennen der Einzelwissenschaften oder des praktischen Lebens ist, macht wenigsteus in dem prinzipiellen Verhältnis zur Erkenntnistheorie keinen wesentlichen Unterschied. Auch der Einzelforscher steht als solcher auf dem „naiven" oder ,natürlichen" Standpunkt. Auch solche Erkenntnistheoretiker, die, wie z. B. Külpe, ausdrücklich bei ihrer Untersuchung die Einzelwissenschaften $\mathrm{zu}$ Grunde legen, um das in ihnen vorgefundene Verfahren der „Realisierung“ zu erforschen,4) setzen daher als Ausgangspunkt das von der erkenntnistheoretischen Reflexion noch nicht berührte Erkennen voraus. Aber auch der erkenntnistheoretisch Reflektierende selbst sieht sich genötigt, immer wieder zu diesem Ausgangspunkt zurückzukehren. $\mathrm{Ob}$ er nun ausdrücklich das Erkennen selbst zum Gegenstand seiner Untersuchuug macht oder in regressivem Verfahren gleichsam hinter dasselbe auf seine Bedingungen zurückgeht: will er nicht ständig unbegründete An-

1) vgl. die Überschrift des I. Abschnitts der „Grundlegung zur Metaphysik der Sitten": Übergang von der gemeinen sittlichen Vernunfterkenntnis zur philosophischen.

2) Ich muss auch hierfür auf mein Buch über „Fries und Kant“ I, $30 \mathrm{ff}$. verweisen.

э) H. Rickert, Zwei Wege der Erkenntnistheorie, Transzendentalpsychologie und Transzendentallogik. Kantstudien XIV, H. 2, S. 4 f.; ders. Der Gegenstand der Erkenntnis, 2. A. (1904) S. $1 \mathrm{f}$.

4) O. Külpe, Die Realisierung, ein Beitrag zur Grundlegung der Realwissenschaften I (1912) S. 2 ff. 
nahmen in seine Theorie hineintragen, so muss er sich immer wieder vergegenwärtigen, was Erkennen, unbeeinflusst von der Theorie von ihm selbst, ist. Und er kann dies; denn so weit er sich auch in seiner Wissenschaft von dem natürlichen Standpunkt entfernt haben mag, im praktischen Leben sieht er sich doch immer im Banne der natürlichen Auffassung, die man auch als ,naiven Realismus" bezeichnet hat, die mạn aber, sofern sie auch für den, der sie wissenschaftlich überwunden hat, im praktischen Leben weiterbesteht, besser als praktischen Realismus bezeichnen würde.

In welcher Weise dieser ,praktische Realismus als Ausgangspunkt", der also nicht bloss die Anfänge, sondern die gesamte erkenntnistheoretische Reflexion begleitet, von dieser selbst bearbeitet wird, haben wir hier nicht weiter zu verfolgen. Wir heben nur zwei Folgerungen hervor, die sich für die in unseren Erörterungen berührten prinzipiellen Fragen ergeben. Erstens, dass die Erkenntnistheorie nicht bloss Psychologie sein kann, da sie sonst gerade die im praktischen Realismus stets sich findende Annahme eines rom Subjekt und allen seinen Vorstellungen unabhängigen Seins als Möglichkeit im voraus ausschalten würde; żweitens, dass für sie die Psychologie - wobei vorläufig unentschieden bleiben mag, ob in der Form z. B. der Husserlschen Phänomenologie oder der Rickertschen Transzendentalpsychologie oder irgend einer empirischen Psychologie - eine umfassende Bedeutung haben muss, da eine Theorie des Erkennens ohne genaue Kenntnis des nach dem praktischen Realismus „im“ Subjekt vor sich gehenden Erkenntnisprozesses nicht denkbar ist. Die Psychologie setzt allerdings die Erkenntnistheorie voraus aber nur in der systematischen Ordnung der Wissenschaft, nicht in ihrem Betrieb, in dem Letzteren schon deshalb nicht, weil ihre wichtigsten Vorgänge, die an das praktische Leben anknüpfenden Vorstellungen, Gefühle, Wollungen nur denselben praktischen Realismus voraussetzen, der auch den Ausgangspunkt der Erkenntnistheorie bildet, und in ihrer Tatsächlichkeit durch keine erkenntnistheoretische: Zerstörung dieses Standpunktes berührt werden. Dagegen bleibt die Anwendung etwaiger erkenntnistheoretischer Ergebnisse auf ihre Erkenntnis als solche ebenso vorbehalten, wie die Erkenntnistheorie selbst, die einem ähnlichen Zirkel verfällt, sich vorbehalten muss, ihre Ergebnisse auf ihre eigene Untersuchung anzuwenden. 
Dieser Vorbehalt setzt allerdings wiederum voraus, dass es einen von der Untersuchung selbst noch unberührten Ausgangspunkt gibt und bestätigt damit die Notwendigkeit, von dem auszugehen, was wir als praktischen Realiswus bezeichnet haben. Für eine erkenntnistheoretische Reflexion, die ohne ein von ihr selbst noch unberührtes "Gegebenes" sich selbst untersuchen wollte, wäre jener Zirkel unüberwindlich.

- Die Erkenntnistheorie als Wissenschaft wird diesen ihren eigenen Ausgangspunkt zerstören, aber sie kehrt doch ständig zu ihm zurück und orientiert sich an ihm. Wie dem Astronomen die scheinbare Bewegung der Himmelskörper, deren Wahrnehmung er mit dem Laien teilt und deren Schein er durchschaut, immer wieder zum Ausgangspunkt und zur steten Orientierung dient, um das Universum wissenschaftlich $\mathrm{zu}$ durchmessen, so muss der Denker von der Höhe seiner Abstraktionen immer wieder zum „natürlichen Weltbegriff" zurückkehren, der ihm, obwohl er seine Unhaltbarkeit durchschaut, nicht bloss in seinen Handlungen der selbstverständliche Schauplatz, sondern auch in seinen tiefsten Forschungen und kühnsten Ideen Ausgangspunkt und Orientierungsmittel bleibt. 